\title{
Application of Compressive Sensing in Cognitive Radio Communications: A Survey
}

\author{
Shree Krishna Sharma, Member, IEEE, Eva Lagunas, Member, IEEE, \\ Symeon Chatzinotas, Senior Member, IEEE and Björn Ottersten, Fellow, IEEE
}

\begin{abstract}
Compressive Sensing (CS) has received much attention in several fields such as digital image processing, wireless channel estimation, radar imaging, and Cognitive Radio (CR) communications. Out of these areas, this survey paper focuses on the application of CS in CR communications. Due to the underutilization of the allocated radio spectrum, spectrum occupancy is usually sparse in different domains such as time, frequency and space. Such a sparse nature of the spectrum occupancy has inspired the application of $\mathrm{CS}$ in $\mathrm{CR}$ communications. In this regard, several researchers have already applied the CS theory in various settings considering the sparsity in different domains. In this direction, this survey paper provides a detailed review of the state of the art related to the application of CS in CR communications. Starting with the basic principles and the main features of $\mathrm{CS}$, it provides a classification of the main usage areas based on the radio parameter to be acquired by a wideband CR. Subsequently, we review the existing CSrelated works applied to different categories such as wideband sensing, signal parameter estimation and Radio Environment Map (REM) construction, highlighting the main benefits and the related issues. Furthermore, we present a generalized framework for constructing the REM in compressive settings. Finally, we conclude this survey paper with some suggested open research challenges and future directions.
\end{abstract}

Index Terms-Cognitive Radio, Compressive Sensing, Wideband Sensing, Radio Environment Map, Compressive Estimation

\section{INTRODUCTION}

Recently, Compressive Sensing (CS), also known as compressive sampling or sparse sampling [1], [2], has been a topic of extensive research in various areas such as digital image processing [3], wireless channel estimation [4], [5], radar imaging [6], Cognitive Radio (CR) [7], electromagnetics [8], etc. Out of the wide range of the aforementioned application areas, this survey paper focuses on the application of CS to CR communications.

Spectrum scarcity is one of the most important challenges faced by today's wireless operators to provide high data rate services to a large number of users. In this context, CR communication has been considered as a potential candidate to address the spectrum scarcity problem in the future generation of wireless communications, i.e., 5G. The concept

Copyright (c) 2015 IEEE. Personal use of this material is permitted. However, permission to use this material for any other purposes must be obtained from the IEEE by sending a request to pubs-permissions@ieee.org.

The authors are with the Interdisciplinary Centre for Security, Reliability and Trust (SnT) (http://www.securityandtrust.lu), University of Luxembourg, Luxembourg-city, L-2721, Luxembourg, email: \{shree.sharma, eva.lagunas, symeon.chatzinotas, bjorn.ottersten\}@uni.lu.

This work was supported by FNR, Luxembourg under the CORE projects "SeMIGod" and "SATSENT". of CR was firstly proposed by J. Mitola in the late 1990's [9] and after its conception, several researchers and industrial/academic/regulatory bodies have been working towards the implementation of this technology. It has a wide range of application areas ranging from Television WhiteSpaces (TVWSs) [10] to satellite communications [11], [12]. The main functions of a CR are to be aware of its surrounding radio environment, i.e., spectrum awareness, and to utilize the available spectral opportunities effectively, i.e., spectrum exploitation.

CS theory states that certain signals can be recovered from far fewer samples or measurements than the samples required by traditional methods [1], [2]. In this approach, a significantly reduced number of measurements is obtained from the incoming data stream and is expected to be reconstructible from these small number of measurements. This method basically combines the following key concepts: (i) sparse representation with a choice of a linear basis for the class of the desired signal, and (ii) incoherent measurements of the considered signal to extract the maximum information using the minimum number of measurements [13]. In sparse signals, most of the signal energy is concentrated in a few non-zero coefficients. Furthermore, to apply the CS theory, it's not necessary for the signal itself to be sparse but can be compressible within sparse representations of the signal in some known transform domain [14], [15]. For example, smooth signals are sparse in the Fourier basis whereas piecewise smooth signals are sparse in the wavelet basis [1].

Although there exist several survey papers in the areas of CR communications covering a wide range of areas such as Spectrum Sensing (SS) [16], spectrum occupancy measurement campaigns [17], spectrum management [18], emerging applications [19], spectrum decision [20], spectrum access strategies [21], CR techniques under practical imperfections [22], and CR networks [23], a comprehensive review on the applications of CS in CR communications is missing from the literature. Besides, there exist several applications of CS in CR communications and they have been investigated for various objectives. In this context, first, this survey paper categorizes the application areas based on the acquired environmental information. Subsequently, it provides a comprehensive review of the existing state of art in these categories. Furthermore, we identify the major issues associated with each of these application areas and present a generalized framework for Radio Environment Map (REM) construction in compressive settings. Finally, we suggest some interesting open research issues and future directions. 
The remainder of this paper is structured as follows: Section II-A provides the basic principles of CS and highlights several important aspects such as uniqueness of a solution and compressive signal processing. Section II-B briefly discusses $\mathrm{CR}$ communications and classifies various application areas of $\mathrm{CS}$ in $\mathrm{CR}$ communications based on the parameter to be acquired. Subsequently, Section III identifies the practical limitations for wideband sensing and reviews in detail the CS-related prior work. Section IV describes the existing approaches for performing the compressive estimation of various signal parameters while Section V discusses various aspects of Radio Environment Map (REM) construction. Finally, Section VI provides open research issues and Section VII concludes this paper. To improve the flow of this paper, we provide the structure of the paper in Fig. 1 and the definitions of acronyms/notations in Table I.

\section{CS AND Its ApPliCATIONS IN CR COMMUNiCATIONS}

In this section, we provide an overview of the basic concepts related to CS theory. The detailed explanation about the fundamental developments in CS can be found in [1], [2], [13].

\section{A. Compressive Sensing Basics}

1) Basic Principle: CS [13], [24], [25] is a novel sensing/sampling paradigm that allows, under certain assumptions, the accurate recovery of signals sampled below the Nyquist sampling limit. In order to briefly review the main ideas of CS, consider the following finite length, discrete time signal $\mathrm{x} \in$ $\mathbb{R}^{L}$. Representing a signal involves the choice of a dictionary, which is the set of elementary waveforms used to decompose the signal. Sparsity of a signal is defined as the number of non-zero elements in the signal under some representation. A signal is said to have a sparse representation over a known dictionary $\boldsymbol{\Psi}=\left[\begin{array}{llll}\boldsymbol{\psi}_{0} & \boldsymbol{\psi}_{1} & \cdots & \boldsymbol{\psi}_{M-1}\end{array}\right]$, with $\boldsymbol{\psi}_{m} \in \mathbb{R}^{L \times 1}$, if there exists a sparse vector $\boldsymbol{\theta}=\left[\begin{array}{llll}\theta_{0} & \theta_{1} & \cdots & \theta_{M-1}\end{array}\right]^{T}$ such that

$$
\mathbf{x}=\sum_{m=1}^{M-1} \boldsymbol{\psi}_{m} \theta_{m} \quad \text { or } \quad \mathbf{x}=\boldsymbol{\Psi} \boldsymbol{\theta}
$$

with $\|\boldsymbol{\theta}\|_{l_{0}}=K<<M$. The $l_{0}$-norm used throughout this paper simply counts the number of non-zero components in $\boldsymbol{\theta}$. A dictionary that leads to sparse representations can either be chosen as a prespecified set of functions or designed by adapting its content to fit a given set of signal examples [26].

The framework of CS aims at recovering the unknown vector $\mathbf{x}$ from an underdetermined system of linear equations

$$
\mathbf{y}=\boldsymbol{\Phi} \mathbf{x}
$$

where $\mathbf{y} \in \mathbb{R}^{\kappa \times 1}$ is the received data vector and matrix $\boldsymbol{\Phi} \in \mathbb{C}^{\kappa \times L}$ with $K<\kappa<L$ is the sub-sampling matrix or sensing matrix since the number of rows is less than the number of columns. Since $\kappa<L$, this system has more unknowns than equations, and thus the system is not invertible. In particular, (2) has infinitely many solutions. Among the infinitely many solutions of (2), we are only interested in the sparsest one. Direct minimization of $\|\boldsymbol{\theta}\|_{l_{0}}$ is an NP-hard problem, which basically means that it requires an exhaustive search and, in general, it is not a feasible approach. Special cases of interest for convexity are all the $l_{p}$-norms for $p \geq 1$. Among them, $l_{1}$-norm is very interesting and popular due to its tendency to sparsify the solution. In this context, Chen et al. [27] stated that a sparse signal $\boldsymbol{\theta}$ can be recovered from only $\kappa=O(K \log (L / K))$ linear non-adaptive measurements by solving the following relaxation

$$
\min _{\boldsymbol{\theta}}\|\boldsymbol{\theta}\|_{l_{1}} \quad \text { subject to } \quad \mathbf{y} \approx \boldsymbol{\Phi} \boldsymbol{\Psi} \boldsymbol{\theta}
$$

where $\|\boldsymbol{\theta}\|_{l_{1}}=\sum_{i}\left|\theta_{i}\right|$. Several methods are available in the literature to solve the optimization problem in (3). The $l_{1}$ minimization is a convex problem and can be recast as a Linear Program (LP) [28]. This is the foundation for the Basis Pursuit (BP) techniques [27], [29], [30]. Alternatively, greedy methods, known as Matching Pursuit (MP), can be used to solve (3) iteratively [31], [32].

2) Uniqueness of a Solution: In general, the relationship between the sensing matrix $(\boldsymbol{\Phi})$ and the signal model $(\boldsymbol{\Psi})$ affects the number of measurements required to reconstruct a sparse signal.

Almost all theory of CS is based on the assumption that $\mathrm{D}=\boldsymbol{\Phi} \boldsymbol{\Psi}$ is the concatenation of two orthogonal matrices. These theories follow the uncertainty principle which states that a signal cannot be sparsely represented both in $\Phi$ and $\Psi$ [33]. This claim depends on the similarity between $\boldsymbol{\Phi}$ and $\boldsymbol{\Psi}$. A rough characterization of the degree of similarity between the sparsity and measurement systems is depicted by the mutual coherence, which is given by

$$
\mu(\boldsymbol{\Phi}, \boldsymbol{\Psi})=\max _{\substack{i, j \\ i \neq j}} \frac{\left|\boldsymbol{\phi}_{i}^{H} \boldsymbol{\psi}_{j}\right|}{\left\|\boldsymbol{\phi}_{i}\right\|_{l_{2}} \cdot\left\|\boldsymbol{\psi}_{j}\right\|_{l_{2}}} .
$$

In other words, $\mathbf{D}$ should have columns $\mathbf{d}_{i}, i=1, \ldots, N$ with small correlations. An explicit example of matrices which have small coherence is the concatenation of the Identity and Fourier matrices. Another suitable way to describe $\mu$ is to compute the Gram matrix $\mathbf{G}=\tilde{\mathbf{D}}^{T} \tilde{\mathbf{D}}$, using matrix $\mathbf{D}$ after normalizing each of its columns $(\tilde{\mathbf{D}})$. The mutual coherence is given in this case by the off-diagonal entry of $\mathbf{G}$ with the largest magnitude.

On the other hand, another criterion for evaluating the quality of CS matrices that are nearly orthonormal, is the Restricted Isometry Property (RIP) introduced in [30], initially called as "uniform uncertainty principle". The RIP is a sufficient condition on $\tilde{\mathbf{D}}$ for exact recovery of a $K$-sparse $\boldsymbol{\theta}$.

The matrix $\tilde{\mathbf{D}}$ satisfies the RIP of order $s \in \mathbb{N}, s<L$, if there exists an isometry constant $0<\delta_{s}<1$ such that

$$
\left(1-\delta_{s}\right)\|\boldsymbol{\theta}\|_{l_{2}}^{2} \leq\|\tilde{\mathbf{D}} \boldsymbol{\theta}\|_{l_{2}}^{2} \leq\left(1+\delta_{s}\right)\|\boldsymbol{\theta}\|_{l_{2}}^{2}
$$

holds for all $s$-sparse vectors, where $\delta_{s}$ is the smallest number satisfying (5). However, working with the RIP condition is much more complex compared to the simple coherence concept since for a given matrix, checking the validity of the RIP condition is an NP-hard problem itself. 


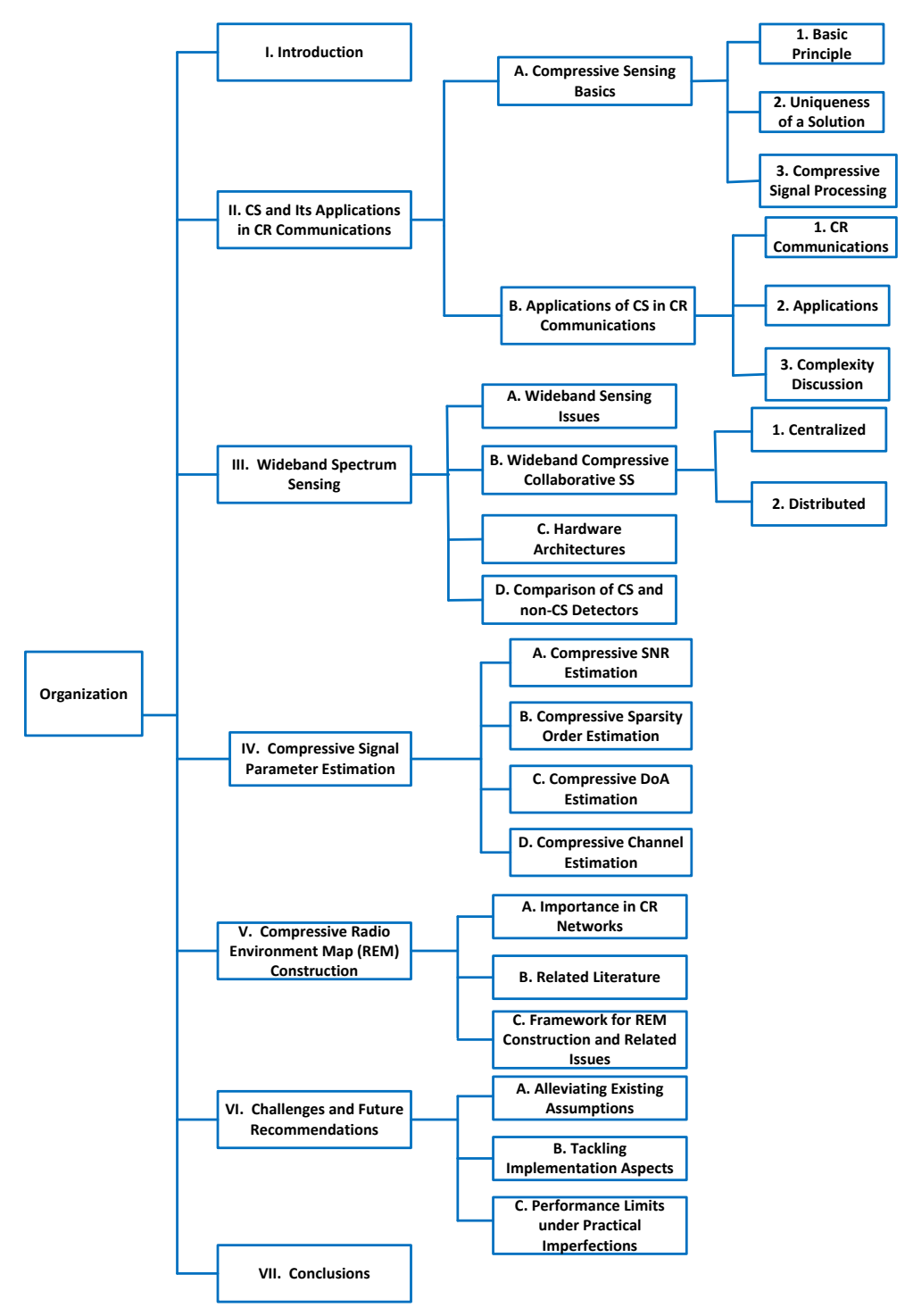

Fig. 1. Structure of the paper

3) Compressive Signal Processing: Many signal processing problems such as detection, estimation, and classification do not require full signal recovery. The CS theory can be further extended to address the detection, estimation and classification problems. In this context, the most relevant works are the discussions of compressive parameter estimation in [34], [35], compressive detection in [36], [42], [43] and compressive classification in [36], [43], [46], [47].

It is possible to apply standard CS to continuous-valued parameter estimation and the detection of signals in continuous domains but it does not perform well due to the discretization of the sparse domain. CS requires the signal to be sparse over a finite basis whereas the parameters/signals could lie anywhere on a continuum. This problem is known in the literature as a basis-mismatch problem [44], [45]. Further, basis-mismatch problems may arise in many other applications including channel estimation discussed later in Section IV-D.

A comprehensive analysis on the performance of signal classification based on compressive measurements is presented in [46]. The first works where sparsity was leveraged to perform classification with very few random measurements are [36], [43], [47]. In particular, [43] focuses on the compressive detection problem but provides some ideas for extensions to classification. Later, [47] explored the use of a compressed version of the matched filter referred to as the smashed filter. The basic idea of the smashed filter is to implement a matched filter directly in the compressed domain without the requirement of reconstructing the original signal from the compressed measurements. The utility of CS projection observations for signal classification by means of an $m$-ary hypothesis testing was proposed in [36]. In general, there are many applications where it can be more efficient and accurate to extract information for classification directly from a signal's compressive measurements than first recover the signal and then extract the information.

\section{B. Applications of CS in CR Communications}

In this section, we provide the basics of CR communication and briefly describe various applications of CS for enabling 
TABLE I

DEFINITIONS OF ACRONYMS AND NOTATIONS

\begin{tabular}{|c|c|c|c|}
\hline Acronyms/Notations & Definitions & Acronyms/Notations & Definitions \\
\hline ADC & Analog to Digital Converter & REM & Radio Environment Map \\
\hline $\mathrm{AIC}$ & Analog to Information Converter & $\mathrm{RF}$ & Radio Frequency \\
\hline BP & Basis Pursuit & RIP & Restricted Isometry Property \\
\hline CDMA & Code Division Multiple Access & SSR & Sparse Signal Representation \\
\hline $\mathrm{CR}$ & Cognitive Radio & SS & Spectrum Sensing \\
\hline $\mathrm{CS}$ & Compressive Sensing & ST & Secondary Transmitter \\
\hline CMUX & Compressive Multiplexer & SVD & Singular Value Decomposition \\
\hline CSI & Channel State Information & SNR & Signal to Noise Ratio \\
\hline DoA & Direction of Arrival & WSS & Wideband Spectrum Sensing \\
\hline DCS & Distributed Compressive Sensing & WSN & Wireless Sensor Network \\
\hline DR & Dynamic Range & QoS & Quality of Service \\
\hline $\mathrm{FC}$ & Fusion Centre & UWB & Ultra-Wideband \\
\hline FFT & Fast Fourier Transform & $\mathbf{x}$ & Nyquist-sampled signal \\
\hline FIR & Finite Impulse Response & $L$ & Number of samples of $\mathbf{x}$ \\
\hline GIS & Geographic Information System & $\Psi$ & Dictionary or sparsifying basis \\
\hline i.i.d. & independent and identically distributed & $\boldsymbol{\theta}$ & Sparse vector \\
\hline JSM & Joint Sparsity Order & $M$ & Number of samples of $\theta$ \\
\hline LP & Linear Program & $\|\boldsymbol{\theta}\|_{l_{p}}$ & $\left(\sum_{m=1}^{M}\left|\theta_{m}\right|^{p}\right)^{1 / p}, p \geq 1$ \\
\hline LASSO & Least Absolute Shrinkage and Selection Operator & $\|\boldsymbol{\theta}\|_{l_{0}}=K$ & number of non-zero components of $\boldsymbol{\theta}$ \\
\hline MP & Marching Pursuit & $\mathbf{y}$ & Compressive-sampled signal \\
\hline MMV & Multiple Measurement Vector & $\kappa$ & Number of samples of $\mathbf{y}$ \\
\hline MWC & Modulated Wideband Converter & $\Phi$ & Sensing matrix \\
\hline NP & Nondeterministic Polymonial & $\bar{D}$ & Product of $\Phi \cdot \Psi$ \\
\hline OMP & Orthogonal Matching Pursuit & $f_{s}$ & Sampling frequency \\
\hline PT & Primary Transmitter & $N_{\text {eff }}$ & Number of ADC effective bits \\
\hline PR & Primary Receiver & $\sum$ & Summation \\
\hline PSD & Power Spectral Density & $(\cdot)^{T}$ & Transpose \\
\hline PU & Primary User & $(\cdot)^{H}$ & Conjugate transpose \\
\hline ISNR & In-band Signal to Noise Ratio & $\mathbb{R}^{\prime}$ & Set of real numbers \\
\hline RSNR & Recovered Signal to Noise Ratio & $\mathbb{C}$ & Set of complex numbers \\
\hline RD & Random Demodulator & $\rho$ & Compression factor \\
\hline RSS & Received Signal Strength & $\odot$ & Element-wise product \\
\hline
\end{tabular}

CR communications. The detailed description on these applications will be provided in latter sections by referring to the current state of the art.

1) $C R$ Communications: Current wireless networks are facing a spectrum scarcity problem due the limited available spectrum and the increasing demand of high data-rate services. On one hand, the usable spectrum seems to be scarce due to spectrum segmentation and the static frequency allocation policy. On the other hand, several spectrum measurement compaigns show the under-utilization of the allocated spectrum in the time and space (geographical) domains [17]. In this context, CR communications has been considered as a promising candidate to address the spectrum scarcity problem in future wireless networks. In CR communications, Primary Users (PUs), also called incumbent or licensed users, are the users who have legacy rights on the use of a specific part of the spectrum. On the other hand, Secondary Users (SUs), also called cognitive or unlicensed users, exploit this spectrum in such a way that they do not provide harmful interference to the normal operation of the licensed PUs.

A CR should be capable of acquiring various Radio Frequency (RF) parameters in order to become aware of its surrounding radio environment. This can be achieved with the help of various spectrum awareness techniques such as Spectrum Sensing (SS), database and the estimation of the signal parameters such as Signal to Noise Ratio (SNR), Channel State Information (CSI), Directional of Arrival (DoA), etc. After being aware of the RF environment, the next important functionality for a $\mathrm{CR}$ is to exploit the available underutilized resource effectively, called spectrum exploitation. The widely used paradigms for spectrum exploitation are interweave, underlay and overlay [48]. Out of these paradigms, the first paradigm consists of interference avoidance/opportunistic techniques which require SUs to communicate opportunistically using the unused spectral holes in different domains such as space, frequency, and time. The second paradigm encompasses interference control/management schemes and allows the coexistence of primary and secondary systems only if the interference caused by Secondary Transmitters (STs) to the Primary Receivers (PRs) can be properly controlled and managed. On the other hand, the third paradigm encompasses advanced coding and transmission strategies at the STs for interference management and may require a higher level of coordination between primary and secondary systems.

2) Applications: Although several contributions exist in the literature dealing with the narrowband CR scenarios, in practice, a CR should be capable of monitoring the surrounding radio environment over a wide spectrum range in order to utilize the benefits of CR communications efficiently. This environmental knowledge over a wideband spectrum helps a CR to apply adaptive resource allocation and spectrum exploitation techniques for the effective utilization of the under-utilized radio spectrum. However, due to the practical limitations on the capability of receiver hardware components, mainly Analog to Digital Converter (ADC), it's difficult to implement wideband spectrum awareness algorithms in practice. This difficulty can be alleviated by utilizing the benefits of CS discussed in Section II.

The RF awareness over a wideband can be acquired mainly with the following mechanisms: (i) Wideband SS, (ii) Signal parameter estimation, and (iii) Database information. The important parameters acquired with these mechanisms are depicted in Fig. 2. In the wideband SS mechanism, the RF parameters to be acquired can be energy level and the power spectrum. Furthermore, in the category of signal parameter estimation, different parameters such as CSI, DoA, SNR and 
TABLE II

EXISTING TECHNIQUES FOR THE APPLICATIONS OF CS IN CR COMMUNICATIONS

\begin{tabular}{|c|c|c|c|}
\hline Awareness Mechanisms & Parameters & Applicable CS techniques & References \\
\hline Spectrum sensing & $\begin{array}{l}\text { Energy level } \\
\text { Power spectrum }\end{array}$ & $\begin{array}{l}\text { Compressive spectrum sensing } \\
\text { Compressive power spectrum estimation }\end{array}$ & $\begin{array}{l}{[7],[49],[50],[52],[53],[59],[63]-[70]} \\
{[71]-[73]}\end{array}$ \\
\hline Signal parameter estimation & $\begin{array}{l}\text { Channel State Information (CSI) } \\
\text { Direction of Arrival (DoA) } \\
\text { Signal to Noise Ratio (SNR) } \\
\text { Sparsity order }\end{array}$ & $\begin{array}{l}\text { Compressive channel estimation } \\
\text { Compressive DoA estimation } \\
\text { Compressive SNR estimation } \\
\text { Compressive sparsity order estimation }\end{array}$ & $\begin{array}{l}{[81],[82]} \\
{[83]-[88]} \\
{[89],[90]} \\
{[91],[127],[147]}\end{array}$ \\
\hline Database information & $\begin{array}{l}\text { Number of active Txs, Tx locations } \\
\text { Transmit power }\end{array}$ & Compressive REM construction & {$[92],[96]-[99]$} \\
\hline
\end{tabular}

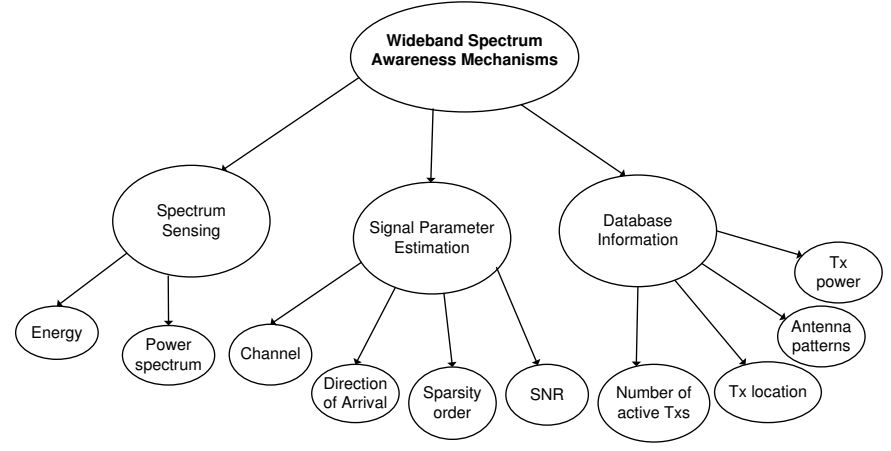

Fig. 2. Wideband spectrum awareness techniques and the main acquisition parameters

sparsity order can be estimated compressively by employing the CS approach. Moreover, in the third category, parameters such as number of active Transmitters (Txs), locations of the active Txs, power levels, etc. can be estimated which are subsequently useful to construct the Radio Environment Map (REM). In Table II, we present various parameters involved with these awareness mechanisms and the related techniques. We further provide the mapping of the related existing techniques with these techniques. The detailed description of these techniques is provided in the subsequent sections.

3) Complexity Discussion: One of the main motivations behind using CS in CR communications is that a CS-based CR transceiver can sense wider spectrum with the same sampling requirements or the same spectrum with reduced sampling requirements, thus resulting in cheaper and more energy efficient systems. However, CS-based receivers are relatively complex due to the involved operations in reconstructing the original sparse signal. For the recovery of the original sparse signals, several recovery algorithms such as Greedy Pursuit, matching Pursuit, Orthogonal Matching Pursuit (OMP), Stagewise Orthogonal Matching Pursuit (StOMP), Gradient Pursuit (GP), Tree-based OMP (TOMP), re-weighted $l_{1}$ minimization, etc. have been proposed in the literature. These algorithms offer different tradeoffs in terms of reconstruction complexity, performance, robustness to noise, as well as the allowable compression ratios for a certain sparsity level of the original sparse signal [37]. Some recovery algorithms are simple to implement, but may require a large number of samples in order to satisfy a desired performance level.

For instance, based on the comparative results presented in [37], the algorithms OMP and TOMP are greedy search algorithms which are fast in computation, however, their recovery accuracy is poor and they need a large number of measurements in order to reach a comparable reconstruction performance to BP and reweighted $l_{1}$ algorithms. On the other hand, $\mathrm{BP}$ and re-weighted $l_{1}$ algorithms provide more accurate solutions but are demanding in terms of computational costs. Thus, in general, there exists a clear tradeoff between the sampling cost and energy saving in computation and it is crucial to balance this tradeoff in order to enhance the overall recovery performance. Another example is that the simple and most commonly used OMP algorithm can be implemented using the following four different methods [38]: (i) naive approach, (ii) Cholesky decomposition, (iii) QR decomposition, and (iv) matrix inversion lemma. These four implementation aspects have different complexities and memory requirements, and depending on the size of the considered problem, any of these four implementations can be the fastest. As the number of samples increases, the computation time of the naive approach becomes much longer than for the other three and for the large problem sizes which require higher number of iterations, the QR decomposition approach appears to be the fastest one [38].

The aforementioned complexity discussion is applicable while carrying out CS-based spectrum sensing using the following steps [7], [69]: (i) acquisition of the compressed samples, (ii) reconstruction of the Nyquist rate signal from the compressed samples, and (iii) spectrum sensing using the reconstructed signal. In this procedure, there have been several attempts to reduce the computational complexity of the employed reconstruction step by utilizing prior information ( [39] and references therein). In this regard, authors in [39] have recently proposed a data-assisted non-iteratively reweighted least squares based CS algorithm by exploiting the prior data obtained from a geo-location database in order to reduce the computational complexity of the previously proposed iteratively re-weighted least squares algorithm [40].

However, as highlighted in Section II-A3, for signal detection/estimation/classification problem in CR applications, it's not necessary to reconstruct the entire original sparse signal. The decision on the presence or the absence of PU signals over the considered spectrum can be made based on the compressed measurements only and the reconstruction step of the commonly used CS technique can be completely illuminated, thus reducing the computational complexity [41], [42]. In this context, authors in [41] proposed a Bayesian formulation to estimate the parameters of the sparse signal directly from the compressed measurements and demonstrated that such a Basysian formulation is computationally less expensive, more accurate, and achieves a higher compression rate compared to 


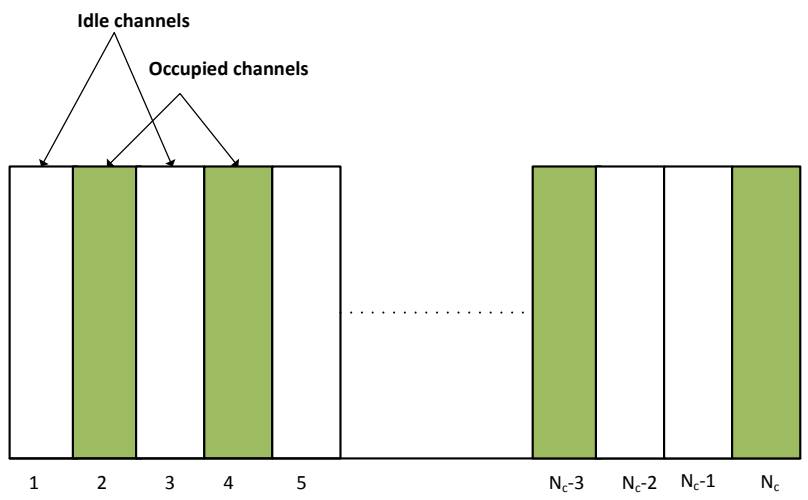

Fig. 3. Schematic representation of a wideband channel with $N_{c}$ number of narrowband channels. Herein, sparsity order is the ratio of the number of occupied channels to the total number of channels.

the traditional non-CS methods such as BP method. Moreover, authors in [42] have shown that in several applications such as detection, estimation and classification, it becomes more efficient and accurate to extract information directly from compressive measurements rather than the traditional approach of first recovering the signal first and then extracting information from the recovered signal.

\section{WidebAnd SPectrum SEnSING}

In CR networks, it is desirable for the SUs to identify spectrum opportunities over a wideband spectrum rapidly and accurately. Figure 3 depicts the schematic representation of a wideband channel with $N_{c}$ number of narrowband channels. As reflected in the diagram, some of the channels are occupied and the remaining are idle at a certain time. In this context, a CR should be able to acquire information about which channels are idle over the considered bandwidth in order to use them in an opportunistic way. For this purpose, an SS technique requires the radio to receive a wideband signal through an RF front-end, sample it by a high speed ADC, and subsequently perform measurements for the detection of the PU signals. For the implementation of wideband SS, a CR transceiver needs to have a wideband antenna, a wideband filter and amplifier, and a high speed ADC. The solutions of wideband antennas and wideband filters are available in the literature [100], [101], however, the development of highspeed ADC technology is lagging [102], [103] due to the challenges involved in building sampling hardware that operates at a sufficiently high rate [104].

The traditional way for detecting spectrum holes over a wideband is to divide the total band into many channels and to perform channel-by-channel sequential scanning [105], which might introduce large latency. Another possible way is to use an RF front-end with a bank of narrow bandpass filters. This approach solves the latency problem since multiple channels can be processed simultaneously. However, it is inefficient to implement due to the requirement of numerous RF components. An alternative approach is to directly sense the wide frequency range at the same time, called Wideband Spectrum Sensing (WSS) (see [106] and the references therein). However, special attention should be paid to the wideband processing which renders high-rate standard ADC costly and even impractical. Clearly, the need to process very wide bandwidth is the most critical challenge for the WSS [107].

To address the aforementioned issues, many researchers have considered CS techniques for wideband SS assuming some sparsity basis. As the wideband spectrum is inherently sparse due to the low percentage of spectrum occupancy, CS becomes a promising technique to reduce the burden on the ADCs in WSS. The important advantage of the CS approach for wideband signal acquisition is that it can increase the overall Dynamic Range (DR) of the acquisition system [49]. In contrast to conventional Nyquist rate sampling systems, CSbased ADCs, also called Analog to Information Converters (AICs) [108] provide an important benefit in reducing the required sampling rate in order to represent the same spectrum. Further, fewer quantization operations are required in CSbased receivers due to the reduction in the number of acquired measurements, thus resulting in significant power savings [109].

Several CS-based approaches have been developed to detect the frequency occupancy of PUs using sub-Nyquist rate samples. CS was first applied to WSS in [7], where sub-Nyquist rate samples are utilized to detect and classify frequency bands through a wavelet-based edge detector. Further, authors in [52] studied a two-step CS scheme with the aim of minimizing the sampling rate, where the actual sparsity was estimated in the first time slot and the compressed measurements were then adjusted in the second slot. In [110], a sequential CS approach has been proposed where each compressed measurement was acquired in sequence. In this sequential CS approach, observations become available sequentially and the process can be stopped as soon as there is a reasonable certainty of correct reconstruction. This approach does not require knowing how sparse is the signal, and allows reconstruction using the smallest number of samples.

The problem of sampling a signal at the minimal rate and reconstructing the original spectrum from the compressive measurements has been discussed in [111]-[113]. Further, power spectrum estimation methods based on sub-Nyquist rate samples were presented in [114], [115], where the spectrum of the uncompressed signal is retrieved by concentrating on the autocorrelation function instead of the original signal itself. Moreover, CS-based correlation matching approaches for identification of the PUs were presented in [116]-[118] in the context of a CR.

In [119], an adaptive SS algorithm, which can adaptively adjust compressed measurements without any sparsity estimation efforts, has been studied. Consequently, the wideband signals are acquired block-by-block from multiple mini-time slots, and gradually reconstruct the wideband spectrum using compressed samples until the spectral recovery is satisfactory. In [49], the performance of a CS-based receiver has been studied with the help of a theoretical analysis of its expected performance with a particular emphasis on noise and DR, and simulation results that compare the CS receiver against the performance expected from a conventional implementation. It has been demonstrated that CS-based systems can potentially 
attain a significantly large DR since they sample at a lower rate. Consequently, it has been shown that CS-based systems that aim to reduce the number of acquired measurements are somewhat sensitive to noise, exhibiting a $3 \mathrm{~dB}$ SNR loss per octave of subsampling similar to the classic noise-folding phenomenon.

The sensing performance of a single node may degrade in wireless channels for several reasons such as the hidden node problem, shadowing, multipath fading, and interference/noise uncertainty. To address these issues, cooperative spectrum sensing, in which several nodes collaborate with each other to enhance the overall sensing performance, has been investigated in several works [120]-[123]. Authors in [123] have compared the performance of soft and hard schemes in which a cooperative node forwards multiple bits of the raw data, i.e., soft cooperative scheme, and a single bit related to the decision on spectrum availability, i.e., hard cooperative scheme, to the fusion center, respectively. By incorporating the reporting interval into the frame structure of a cooperative node and independently of the employed local sensing technique, it has been shown that the hard cooperative scheme provides better performance than the soft cooperative scheme for short sensing times and/or a large number of cooperative nodes. In this particular example, compressive sensing can provide benefits while sensing multiple channels over a wider bandwidth by increasing the dynamic range of the ADC and also in reducing the number of cooperative nodes while sensing multiple number of channels [64]. In the case of a soft cooperative scheme, the CS further helps to reduce the cooperative burden as well as the number of cooperative nodes and in the hard cooperative scheme, the CS is more useful for local sensing. Several works exist in the literature in the context of applying CS for cooperative sensing in centralized [64], [68], [71] and distributed [67], [69], [83], [124] settings. In Section III-B1 and Section III-B2, we provide a detailed discussion on the application of CS in centralized and distributed cooperative SS by referring to the current state of the art.

In the following, we present the main wideband sensing issues, the existing works related to wideband compressive collaborative SS and the hardware architectures.

\section{A. Wideband Sensing Issues}

1. Dynamic Range and Noise Folding: Dynamic Range (DR) describes the range of the input signal levels that can be reliably measured at the same time. In other words, it's the ability to accurately measure small signals in the presence of the large signals. The DR is a useful parameter for any measurement/acquisition system and it is determined by the following two independent parameters [125]: (i) limitation by noise and (ii) limitation by spurious signals.

The DR is defined as the ratio of the full scale amplitude to the peak noise floor and for an $N_{b}$ bit ADC, it is given by

$$
\mathrm{DR}=6.021 N_{b}+1.763 \mathrm{~dB} .
$$

The above equation is valid only in the time domain without digital filtering and a different expression is needed to define the real achievable dynamic range of the system. For a simple acquisition system without a preamplifier, the DR is mainly limited by the $\mathrm{ADC}$ and the $\mathrm{DR}$ in $(\mathrm{dBFS} / \sqrt{\mathrm{Hz}})$ can be written as [125]

$$
\mathrm{DR}=\mathrm{SNR}+10 \times \log \left(f_{s} / 2\right),
$$

where the SNR is given by

$$
\mathrm{SNR}=6.02 \times N_{\mathrm{eff}}+1.76,
$$

where $f_{s}$ is the sampling frequency, $N_{\text {eff }}$ is the number of ADC effective bits.

From practical perspectives, the important advantage of CS for wideband signal acquisition is that it can increase the overall DR of the acquisition system as compared to the conventional Nyquist rate acquisition system within the same instantaneous bandwidth. Due to this advantage, it can reduce the system size, weight, and power consumption, and the monetary cost considerably but at the cost of increasing the noise figure of the system. The exact value of the DR improvement that can be achieved depends on the exact speed and the exact ADC design. Generally, CS-enabled sampling rate reduction can increase the system DR, approximately by one bit (approx. by $6 \mathrm{~dB}$ ) for every factor of 2 that CS permits the ADC sampling rate to be reduced [126].

If (i) the noiseless input is sparse, (ii) the additive noise is white, and (iii) the CS measurement process satisfies the RIP, then the Recovered SNR (RSNR) is related to the In-band SNR (ISNR), which measures the SNR by including only the noise within the same bandwidth as the signal [49], of the received signal in the following way [126]

$$
\rho \frac{1-\delta}{1+\delta} \leq \frac{\mathrm{ISNR}}{\mathrm{RSNR}} \leq \rho \frac{1+\delta}{1-\delta}
$$

where $\rho$ is the compression factor (decimation rate) and $\delta \in(0,1)$ is a constant determined by the CS measurement process. The value of $\rho$ must be less than a critical value $\rho_{C}=B / W$ ( $B$ being the instantaneous bandwidth and $W$ being the maximum signal bandwidth) i.e., the degree of sparsity of the input signal. The above ratio can also be written as [49]

$$
\frac{\mathrm{ISNR}}{\mathrm{RSNR}} \approx 10 \log _{10}(\rho) .
$$

From (10), it can be deduced that every time we double the compression factor $\rho$ (i.e., a one octave increase) up to the value of $\rho_{C}$, the RSNR of the recovered signal decreases by $3 \mathrm{~dB}$. This $3 \mathrm{~dB} /$ octave SNR degradation depicts an important tradeoff while designing CS-based receivers. The main conclusion is that for a fixed signal bandwidth $W / 2$, there is a practical limit to the instantaneous bandwidth $B / 2$ for which we can obtain a desired RSNR [126]. Although the above noise folding behavior of CS systems imposes a very real cost, the dominant advantage is that it increases the DR of the acquisition system.

2. Sampling Rate and Sparsity Order: To determine a suitable sampling rate, most existing works implicitly assume that the sparsity order of the underutilized spectrum is known beforehand. However, in practical CR applications, the actual sparsity order level corresponds to the instantaneous spectrum occupancy of wireless users which is time varying in nature. 
Thus, the actual sparsity level is often unknown and only its upper bound, which can be measured from the maximum spectrum utilization observed statistically over a time period, can be obtained. Hence, in practice, the conservative determination of the sampling rate based on its upper bound can cause unnecessarily high acquisition costs [104].

From the above discussion, it can be noted that the sampling rate depends on the sparsity level and we need to adapt the CS system in such a way that the sampling rate is adaptive in accordance with the dynamic variation of the spectrum occupancy. One method of addressing this aspect to estimate the sparsity order first and then apply the suitable sampling rate based on the estimated sampling rate. In this context, the authors in [127] have proposed a twostep CS approach in which the sparsity order is estimated at the first step by considering sufficiently smaller number of measurements and then the sampling rate corresponding to the estimated sparsity order is applied at the second step to collect additional samples. Subsequently, the reconstruction of the signal spectrum has been carried out using all the collected samples in both steps. Finally, based on this reconstructed signal spectrum, SS decision is made. The aspects of sparsity order estimation are highlighted later in Section IV-B.

Another main benefit of CS-based CR transceiver is that the reduction in the sampling rate of an ADC due to $\mathrm{CS}$ directly translates into the power savings and it becomes more power efficient solution than the traditional non-CS based transceivers. The power consumed by an ADC increases at a rate of $1.1 f_{s}$, where $f_{s}$ is the sampling rate of the ADC. For example, an 8-bit flash ADC at 200 Msps consumes 2320 $\mathrm{mW}$ of power (or $11.6 \mathrm{~nJ} / \mathrm{sample}$ ), while an 8-bit flash ADC at $20 \mathrm{Msps}$ consumes only $150 \mathrm{~mW}$ (or $7.5 \mathrm{~nJ} / \mathrm{sample)} \mathrm{[109].}$ Therefore, in this example, by reducing the sampling rate by a factor of 12.5 , one can reduce the power consumption approximately by a factor of 15.5 .

\section{B. Wideband Compressive Collaborative SS}

As mentioned earlier, WSS is challenging due to the requirement of complex and costly hardware circuitry at the cognitive transceiver. One possible way to address this issue is to perform collaborative SS among the CR nodes in compressive settings which can improve the ability of monitoring over the whole available spectrum band [64], [65] and also can enhance the accuracy of the acquired information. In this context, collaborative compressive SS has been widely studied in the literature utilizing the efficient sampling that exploits the underlying sparse structure of the measured radio spectrum. However, to have the effective realization of the collaborative $\mathrm{CS}$ in a CR network, the following main challenges need to be addressed [65]

1) Conventional cooperation schemes require a Fusion Center (FC) in order to collect measurements from all CRs and to make the centralized sensing decision. This may incur high overhead costs for the reporting links and render the entire network vulnerable to the node failure.

2) The spatially separated cooperating CRs may not be ideally synchronized to remain silent during the SS

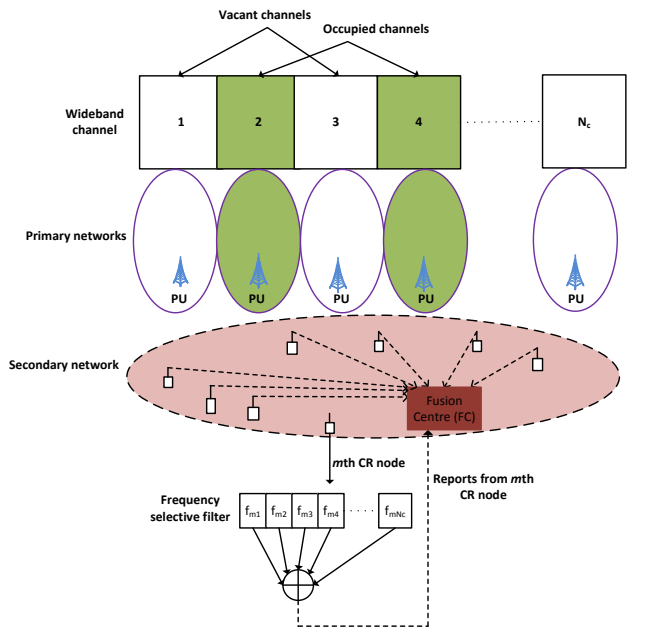

Fig. 4. Illustration of the centralized compressive collaborative SS approach in [64], [129]

phase. Due to this, each CR may perceive not only the common spectral components from the PUs but also individualized spectral innovations arising from the emissions of other CRs or interference in its local onehop region. These CR-dependent spectral innovation components may make the cooperation among the CRs.

The existing literature basically deals with the following two cooperative approaches: (i) centralized and (ii) distributed, which are detailed in the following subsections.

1) Centralized: The centralized approach involves an FC in order to collect the measurements from the spatially separated CRs and a suitable technique is applied at the FC in order to process the collected measurements. In the context, the contribution in [64] studied the centralized compressive cooperative approach in which each CR node senses the linear combinations of multiple channel information and reports them to the FC. Subsequently, the occupied channels are decoded at the FC from the collected reports by using a matrix completion and a joint sparsity recovery algorithms.

In most centralized studies, it is assumed that the FC receives and combines all $\mathrm{CR}$ reports assuming idle reporting channels. However, the reports sent by the CRs are subject to multipath fading and shadowing loss, and thus the entire report data set may not be available at the FC. Further, it may be the case that there are only a few CR nodes in a large network, and thus are unable to gather enough sensing information. In this context, each CR node can be assumed to be equipped with a frequency selective filter, which linearly combines the multiple channel information. Subsequently, these linear combinations are sent as reports to the FC, where the occupied channels are decoded from the reports utilizing suitable CS algorithms.

Figure 4 illustrates the aforementioned centralized collaborative compressive approach studied in [64]. By following this approach, both types of overheads, i.e., the amount of channel sensing at the CRs and the number of reports sent from the CRs to the FC, can be significantly reduced [64]. The two compressive collaborative SS approaches proposed in [64] are 
briefly described below.

1) Matrix completion problem: The aim of this method is to reconstruct a matrix (typically low-rank) efficiently from a relatively small number of observed entries which can be considered as the linear combinations of the channel powers. Each $\mathrm{CR}$ node equipped with $p$ frequency-selective filters takes $p$ linear combinations of channel powers and reports them to the FC. The total $\mathrm{pm}$ linear combinations taken by $m$ CRs form a pm matrix at the FC. This matrix becomes incomplete while incorporating the transmission loss and has the properties enabling its reconstruction only from a small number of its entries. Therefore, information about the complete spectrum usage can be recovered from a small number of reports from the $\mathrm{CR}$ nodes, thus reducing the sensing and communication overloads significantly. Two important properties of a matrix required to apply the matrix completion problem are [128]: (i) low rank, and (ii) incoherence property.

2) Joint sparsity recovery: This method relies on the fact that the spectrum usage information collected by the $\mathrm{CR}$ nodes contains a common sparsity pattern i.e., each of the few occupied channels is typically observed by multiple CRs. Let us represent the sensing information gathered by $M$ CRs in $N$ channels by an $N \times M$ matrix $\mathbf{X}$, where each column corresponds to the channel occupancy status received by the $m$ th $\mathrm{CR}$ and each row represents the occupancy status of the $n$th channel. Since there are only a few number of occupied channels at a time and only a few CRs collect information about a single channel, the matrix $\mathbf{X}$ is jointly sparse without considering the effect of the noise. However, $\mathbf{X}$ can be considered to be approximately jointly sparse while taking the noise into account [64]. There exist different joint sparsity recovery algorithms as described later in Section III-B2 and are applicable to both centralized and distributed CS scenarios.

Similarly, authors in [71] have studied a centralized approach where each sensor collects sub-Nyquist rate samples and forwards them to the FC together with the CSI and the sampler coefficients. Subsequently, the FC calculates the cross-spectra between all measurements and then the power spectrum of the received signals is estimated by exploiting the wide sense stationary property of the PU signals. Furthermore, authors in [68] propose an adaptive sequential CS approach to recover spectrum holes and further propose several fusion techniques to apply the proposed approach in a collaborative manner.

2) Distributed: Distributed CS (DCS) is considered a powerful technique of distributed signal processing in many applications such as sensor networks due to its capability of simultaneous sensing and compression [66]. The theory of DCS relies on the concept of the joint sparsity of a signal ensemble and it exploits both intra- and inter-signal correlation structures [130]. In a typical DCS approach, a number of sensors measure signals (of any dimension) which are each individually sparse in some basis and also may be correlated from sensor to sensor. Each sensor independently encodes its signal by projecting it onto another, incoherent basis (such as a random one) and then transmits just a few of the resulting coefficients to the FC. Subsequently, the FC can jointly reconstruct all of the signals precisely exploiting the joint sparsity of the signal ensemble. In this context, the following two joint sparsity models have been proposed in order to study the DCS problem [130].

- Joint Sparsity Model 1 (JSM-1): In this model, all the signals share a common sparse component while each individual signal contains a sparse innovation component. If $x_{j} \in \mathcal{R}^{N}$ denotes the $j$ th signal in a signal ensemble, with $j \in 1, \ldots, M, M$ being the number of signal sources, i.e., CRs, this model implies the following

$$
x_{j}=z+z_{j}, j \in 1, \ldots, M,
$$

where the signal $z_{j}$ is common to all of the $x_{j}$ with the $K$ sparsity level in the basis $\boldsymbol{\Psi}$, and the signals $z_{j}$ are the unique portions of the $x_{j}$ having $K_{j}$ sparsity level in the same basis. A wireless sensor network used for recording temperature, light intensities, air pressure, etc. can be considered as an example for this model.

- Joint Sparsity Model 2 (JSM-2): This model assumes all signals being constructed from the same sparse set of basis vectors, but with different coefficients, i.e., each measurement vector independently encodes the sparse signals while the ensemble of sparse signals share a common sparsity structure. This model implies the following

$$
x_{j}=\boldsymbol{\Psi} \theta_{j}, j \in 1, \ldots, M,
$$

where each $\theta_{j}$ is supported only on the same $\omega \subset$ $\{1, \ldots, N\}$ with $|\omega|=K$. In other words, all signals are $K$ sparse and are constructed from the same $K$ elements of $\Psi$, but with arbitrarily different coefficients. This model is applicable to the scenarios such as acoustic localization/DoA estimation [83], array processing etc., where multiple sensors acquire the same signal but with phase shifts and attenuations caused by signal propagation.

Authors in [69] studied the distributed compressive SS problem by using the JSM-2 model considered in [130]. Different CR sensing receivers acquire the same wide-band signal from the licensed system at different SNRs and the autocorrelation vectors of the compressed signal from the CRs are collected at the FC. Subsequently, the distributed JSM2 model has been used to obtain an estimate of the signal spectrum. Similarly, the correlation between the measurements of different CRs may be utilized by using a Kronecker product matrix as a basis, called Kronecker sparsifying basis [67]. This basis helps to exploit the two dimensional sparse structure in the measured spectrum using the collaborative measurements taken by several spatially separated CRs. In this context, authors in [67] proposed a modified JSM-2 (JSM2M) model which relaxes the assumptions of the the original JSM1 and JSM-2 models and generates signals which have a common sparse support in the frequency domain with different amplitudes plus innovations due to the hidden PU problem. 
In [124], two cooperative distributed wideband SS approaches for a CR network are proposed utilizing the CS technique. The first approach jointly estimates the spectrum of the PUs based on the compressive measurements obtained by the individual CRs where CSI is assumed to be available. In the second method, each CR user individually recovers the spectrum of the received faded signal without the availability of CSI and makes a local decision on the frequency occupancy of the PU signal based on this spectrum estimate. Subsequently, all CR users collaboratively make a global decision on the frequency occupancy by using a consensus algorithm based on single-hop communication. Recently, authors in [83] have proposed an Multiple-Sensing-Matrices-FOCUSS (MSM-FOCUSS) algorithm for distributed CS and wideband DoA estimation.

\section{Hardware Architectures}

Several hardware architectures have been proposed and implemented in the test-bed environments enabling the compressive samples to be acquired in practical settings. Some of the widely discussed architectures are briefly described below. In Table III, we provide their advantages, disadvantages and the related references.

- Random Filtering: In this method, first, a sparse/compressible signal is captured by convolving it with a random-tap Finite Impulse Response (FIR) filter, and then the filtered signal is downsampled to obtain a compressed signal. The random taps of the filter can be generated using (i) the $\mathcal{N}(0,1)$ distribution or (ii) the Bernoulli/Rademacher distribution of $\{ \pm 1\} \mathrm{s}$. This method is generic to summarize many types of compressible signals and can be applied to streaming as well as continuous-time signals but the number of taps must be known in order to recover the signal from the compressed data [50].

- Random Convolution: In this method, the measurement process consists of convolving the signal of interest with a random pulse followed by random subsampling [131]. This procedure is random enough to be universally incoherent with any fixed representation system, but structured enough to allow fast computations with the help of Fast Fourier Transform (FFT) operations. Random convolution has the following two advantages compared to the completely random strategies: (i) available implicit algorithms based on the FFT, and (ii) many physical systems take observations of a known (and controllable) pulse with an unknown signal i.e., radar imaging.

- Random Demodulator (RD): In this method, a signal is demodulated by multiplying it with a high-rate pseudonoise sequence, which smears the tones across the entire spectrum [59]. Subsequently, a low-pass antialiasing filter is applied and the signal is captured by sampling it at a relatively low rate. The demodulation process ensures that each tone has a distinct signature within the passband of the filter. Since there are only a few tones present in many applications such as in CR networks, it is possible to identify the tones and their amplitudes from the low-rate samples. The main advantage of this approach is that it bypasses the need for a high-rate ADC, thus allowing the use of robust, low-power and readily available components. However, this benefit comes at the cost of highly non-linear reconstruction process, i.e., the need of additional digital processing.

- Modulated Wideband Converter (MWC): In 2010, Mishali et al. proposed an architecture, called MWC, which generally comprises of a bank of modulators and low-pass filters. This architecture first multiplies the analog signal by a periodic waveform, whose period corresponds to the multiband model parameters. A squarewave alternating at the Nyquist rate is one choice; other periodic waveforms are also possible. Subsequently, the product is lowpass filtered and sampled uniformly at a low rate. The goal of the modulator is to alias the spectrum into baseband. The most distinguishing characteristic of the MWC from that of the RD is that the RD has sampling functions that have finite temporal extent but infinite spectral support while the MWC employs sampling functions that have finite spectral support but infinite temporal support [138].

- Compressive Multiplexer (CMUX): This method does not require the calibration of an analog low-pass filter or integrator as required in the random modulator and MWCs. Furthermore, the basic calibration in this method can be simply achieved with the knowledge of a few resistor values and unlike other parallel structures, it requires only one $\mathrm{ADC}$ rather than one per channel [53]. It can be considered to be analogous to coded digital communications schemes such as Code Division Multiple Access (CDMA). Instead of coding the signal with orthogonal codes and transmitting into the same channel, the CMUX effectively codes each channel with a near orthogonal code and then combines the coded channels together following the approach proposed in [53]. Recently, authors in [54] have proposed a CMUX architecture for the acquisition of the ensembles of correlated signals by exploiting the correlation structure of the signal ensemble even though it is unknown a priori.

\section{Comparison of CS and non-CS Detectors}

Spectrum sensing in a CR involves deciding whether the PU is present or not from the observed signals. Thus, spectrum sensing can be formulated as a binary hypothesis testing problem in the following way

$$
y(n)= \begin{cases}w(n) & \mathcal{H}_{0}, \\ s(n)+w(n) & \mathcal{H}_{1}\end{cases}
$$

where $y(n)$ denotes the received signal at the CR device at the $n$th sampling instance, $s(n)$ denotes the primary signal and $z(n)$ is the Additive White Gaussian Noise (AWGN). The CR user has to decide if the primary signal is present $\left(\mathcal{H}_{1}\right)$ or not $\left(\mathcal{H}_{0}\right)$ from the observations $y(n)$ collected over the sensing duration. 
TABLE III

ADVANTAGES AND DISADVANTAGES OF THE EXISTING COMPRESSIVE ARCHITECTURES

\begin{tabular}{|c|c|c|}
\hline Name and References & Advantages & Disadvantages \\
\hline $\begin{array}{l}\text { Random Filtering (RF) } \\
{[50],[51]}\end{array}$ & $\begin{array}{l}\text { Applicable to many types of compressible signals } \\
\text { Measurement operator can be stored and applied efficiently } \\
\text { Easy implementation }\end{array}$ & $\begin{array}{l}\text { Number of filter taps must be known } \\
\text { Nonlinear reconstruction algorithm }\end{array}$ \\
\hline $\begin{array}{l}\text { Random Convolution (RC) } \\
\text { [131], [132] }\end{array}$ & $\begin{array}{l}\text { Available implicit algorithms based on the FFT } \\
\text { Utilization of the known pulse in many physical systems }\end{array}$ & $\begin{array}{l}\text { Not applicable for all sparse/compressible signals } \\
\text { The pulse structure may not be known }\end{array}$ \\
\hline $\begin{array}{l}\text { Random Demodulator (RD) } \\
\text { [59], [60], [62] }\end{array}$ & $\begin{array}{l}\text { No need for a high-rate ADC } \\
\text { Robust against noise and quantization errors }\end{array}$ & $\begin{array}{l}\text { Slow reconstruction process and high sampling delay } \\
\text { Only suitable for signals having a finite set of pure sinusoids }\end{array}$ \\
\hline $\begin{array}{l}\text { Modulated Wideband Converter (MWC) } \\
\text { [61], [63] }\end{array}$ & $\begin{array}{l}\text { Suitable for analog multiband signals } \\
\text { Parameter choice is insensitive to the exact bandwidth } \\
\text { Flexible control of sampling rate at each channel } \\
\text { Fast reconstruction process and low sampling delay }\end{array}$ & $\begin{array}{l}\text { Requires ideal low pass filters for reconstruction } \\
\text { Imperfections of non-ideal lowpass filters } \\
\text { Limited number of bands and bandwidth }\end{array}$ \\
\hline $\begin{array}{l}\text { Compressive Multiplexer (CMUX) } \\
\text { [53], [54] }\end{array}$ & $\begin{array}{l}\text { It requires only one ADC rather than one per channel } \\
\text { Flexibility to increase the total bandwidth } \\
\text { Simpler calibration }\end{array}$ & $\begin{array}{l}\text { Undersampling factor is more restricted } \\
\text { Inherent non-idealities in the RF tuner }\end{array}$ \\
\hline
\end{tabular}

In compressive settings, the above detection problem can be written in the following way

$$
\mathbf{y}= \begin{cases}\mathbf{\Phi} \mathbf{w} & \mathcal{H}_{0}, \\ \mathbf{\Phi}(\mathbf{s}+\mathbf{w}) & \mathcal{H}_{1},\end{cases}
$$

where $\mathbf{y}$ is a $\kappa \times 1$ compressive-sampled received signal, $\mathbf{\Phi}$ is a $\kappa \times L$ compressive matrix, $\mathbf{s}$ is an $L \times 1$ PU signal vector, and $\mathbf{w}$ denotes the $L \times 1$ AWGN vector. If we already know the value of $\mathbf{s}$ during the design of $\boldsymbol{\Phi}$, the optimal strategy is to choose the value of $\boldsymbol{\Phi}$ as $\boldsymbol{\Phi}=\mathbf{s}^{T}$. However, since this knowledge is difficult to obtain in practice for the case of CR applications, the value of $\boldsymbol{\Phi}$ should be universal and is considered to be a random matrix in most of the existing literature [42].

With regard to the detection problem (13), there exist several CR techniques in the literature. The main SS techniques are matched filter based detection, Energy Detection (ED), feature-based detection, autocorrelation based detection, covariance based detection, eigenvalue based detection, etc [16], [22], [55]. Corresponding to the hypothesis testing problem in compressive settings represented in (14), a general framework for signal processing of compressed measurements for detection and estimation without reconstructing the original signal has been detailed in [42]. A much more involved analysis for the estimation setting was presented in [58], where the behavior of the achievable estimation performance in the sparse setting has been analyzed. Out of the aforementioned SS techniques, in this paper, we analyze the performance of the following detectors in compressive and non-compressive settings.

- Matched Filter Detection: The matched filter is an optimal detector in the presence of stationary Gaussian noise since it maximizes the received SNR. However, it requires a priori knowledge of the primary signal and the performance may degrade if this information is not accurate. In practice, most wireless systems have pilots, preambles, synchronization words or spreading codes that can be used for the coherent detection.

- Energy Detection: The energy detector is the most common way of spectrum sensing because of its low complexity (computational and implementation) [56]. It can be considered as a semiblind technique since it only requires the knowledge of the noise variance and does not rely on any signal feature. The main drawback of the energy detector is its inability to discriminate between sources of received energy (the primary signal and noise) making it susceptible to uncertainties in background noise power, specially at a low SNR.

- Feature-based Detection: If some features of the primary signal such as its carrier frequency or modulation type are known a priori, more sophisticated feature detectors may be employed to carry out spectrum sensing at the cost of increased complexity. Cyclostationary detection [57] and correlation matching detection [116]-[118] are particularly appealing because of their ability to distinguish the primary signal from the interference and noise. They can work in a very low SNR region due to their noise rejection capability but sometimes they are computationally complex and requires significantly long observation time.

Next, we present some numerical results about the performance of the aforementioned three types of SS techniques. To analyze the performance in compressive settings, we consider a multi-coset sampling (periodic non-uniform sampler) in which the total number of received samples is divided into blocks, and the same compressive matrix $\boldsymbol{\Phi}$ is applied to each block. Figure 5 depicts the probability of detection $\left(P_{d}\right)$ versus SNR results for a primary signal in AWGN considering a fixed probability of false alarm $P_{f}=10^{-3}$. From the figure, it can be noted that the matched filter outperforms the simple energy detector since it is able to reliably detect low-power primary signals. The value of $\rho$ in Fig. 5 indicates the compression ratio, i.e., the ratio of the number of rows to the number of columns in $\boldsymbol{\Phi}$, defined in Section II, and the value $\rho=1$ represents the Nyquist rate sampling, i.e., the conventional non-CS approach. As the value of $\rho$ decreases, i.e., we use more compression, the detection performance of both matched filter and the energy detector with respect to SNR decreases as depicted in Fig. 5. This means that there exists a clear tradeoff between the detection performance and the sampling rate for both matched filter and the energy detector.

In addition, we provide the performance comparison of correlation matching detectors (which falls under the category of feature-based detection [117]) in CS and non-CS settings in Fig. 6. The scenario considers a desired Binary Phase Shift Keying (BPSK) signal with SNR $=10 \mathrm{~dB}$ at the normalized frequency of 0.2 and a pure-tone interference with $\mathrm{SNR}=10 \mathrm{~dB}$ located at the normalized frequency of 


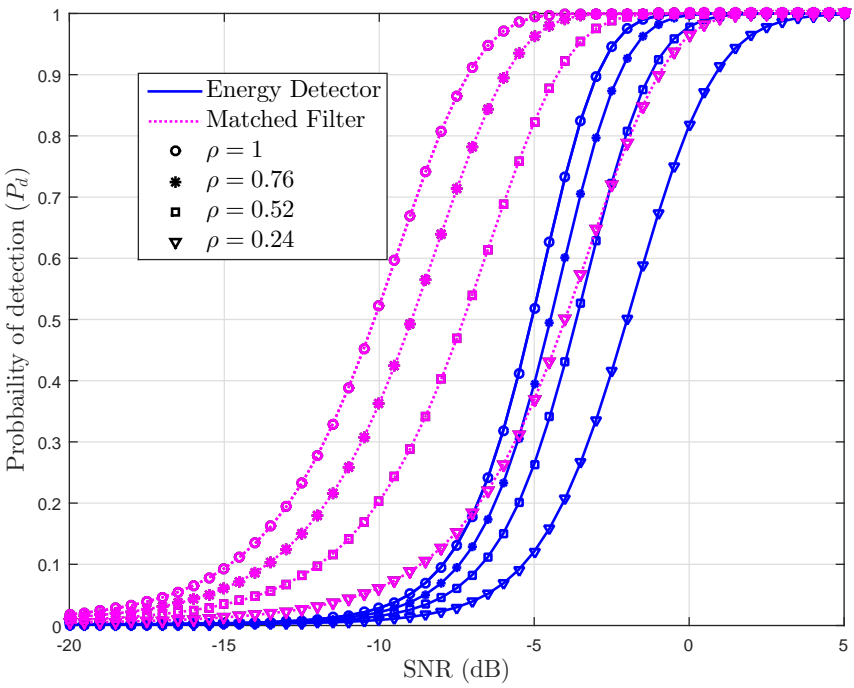

Fig. 5. Probability of detection versus SNR of the energy detector and matched filter with compressive measurements (Probability of false alarm, $P_{f}=10^{-3}$ ), Number of Nyquist rate samples $=99$.

0.7. Figure 6(a) uses the Euclidean metric (Frobenius norm) which works as a conventional energy detector, and it can be noted that this approach does not provide a good performance in discriminating interference from the desired signal. On the other hand, the result in Fig. 6(b) uses the minimum eigenvalue technique presented in [117], [118] and this method is able to distinguish the desired signal from the interference effectively. Furthermore, the presented results in Figs. 6(a) and 6(b) show the degradation of the correlation matching-based WSS techniques in terms of the capability of distinguishing the desired signal from the interference with the decrease in the compression ratio $\rho=1$, i.e., with more compression. However, in Fig. 6(b), it is interesting to note that the power level estimation does not suffer due to compression since the main peak is located at the true frequency with the level close to the SNR value of $10 \mathrm{~dB}$.

\section{Compressive Signal PARAmeter Estimation}

As described in Section II-B, a CR may acquire different signal parameters such as SNR, channel, sparsity order, etc. for enabling $\mathrm{CR}$ communications. In contrast to the most commonly used spectrum occupancy information required for an interweave CR, the parameters such as SNR, DoA, CSI, etc. will allow the CR to implement underlay CR techniques such as cognitive beamforming [139], cognitive interference alignment [140], Exclusion Zone (EZ), and power control [141]. Due to the practical constraints in the acquisition hardware, the CS-based approach can be utilized to estimate these parameters compressively, leading to the saving in the hardware resources [34]. In the following, we describe the existing contributions which utilized the CS approach in order to acquire these parameters.

\section{A. Compressive SNR Estimation}

In the existing literature, various data-aided and non dataaided SNR estimators have been investigated in the context

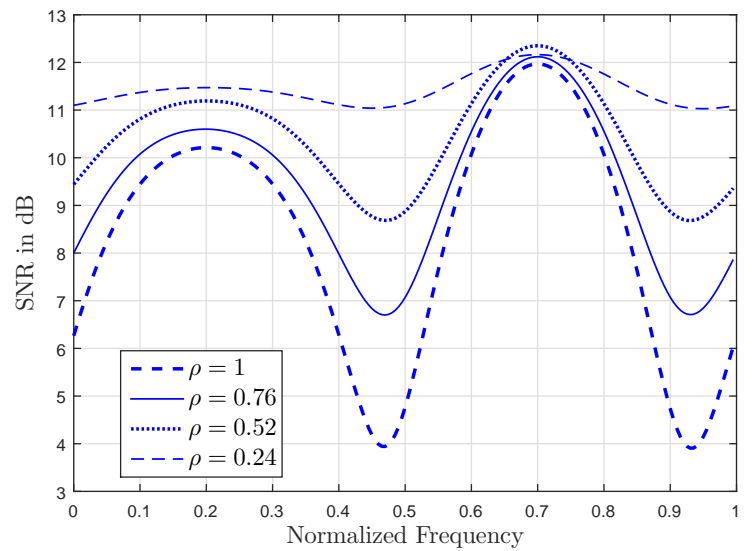

(a)

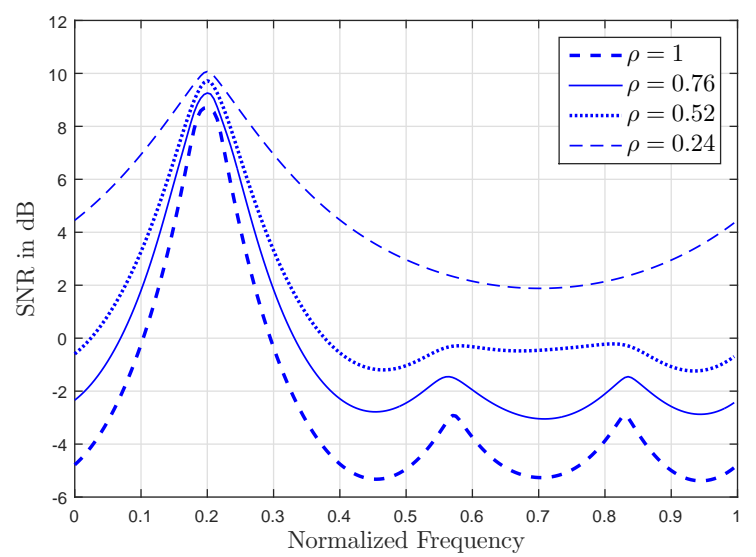

(b)

Fig. 6. Performance of CS-based correlation-matching wideband detector [117], [118]. In the considered scenario, there is a desired BPSK signal with SNR $=10 \mathrm{~dB}$ at the normalized frequency of 0.2 and there is a pure-tone interference located at the normalized frequency of 0.7 and with $\mathrm{SNR}=10 \mathrm{~dB}$. The parameter $\rho$ defines the compression rate/ratio with $\rho=1$ indicating the Nyquist rate sampling, i.e., no compression. (a) detector based on the traditional Euclidean metric (Frobenius norm), (b) detector based on the minimum eigenvalue technique proposed in [117], [118].

of traditional legacy based systems (see [142] and reference there in). SNR estimation is helpful for legacy based systems in order to implement adaptive techniques such as handoff algorithms, adaptive bit loading and optimal soft value calculation for improving the performance of channel decoders. In addition to the aforementioned benefits, estimation of primary SNR is useful for CR-based systems in order to implement proper underlay transmission strategies [89].

Existing SNR estimation literature mostly focus on narrowband CR systems [89], [143]-[145] where the application of CS does not provide much benefit. However, in practice, it is highly desirable to estimate the primary SNR over the wideband spectrum in order to utilize the available spectrum opportunities effectively. In this context, authors in [90] recently studied an eigenvalue-based compressive SNR estimation problem for a wideband cognitive receiver utilizing the CS approach. The following two correlated scenarios have been studied considering the equal received power across all the carriers: (i) correlated noise, and (ii) correlated Multiple 


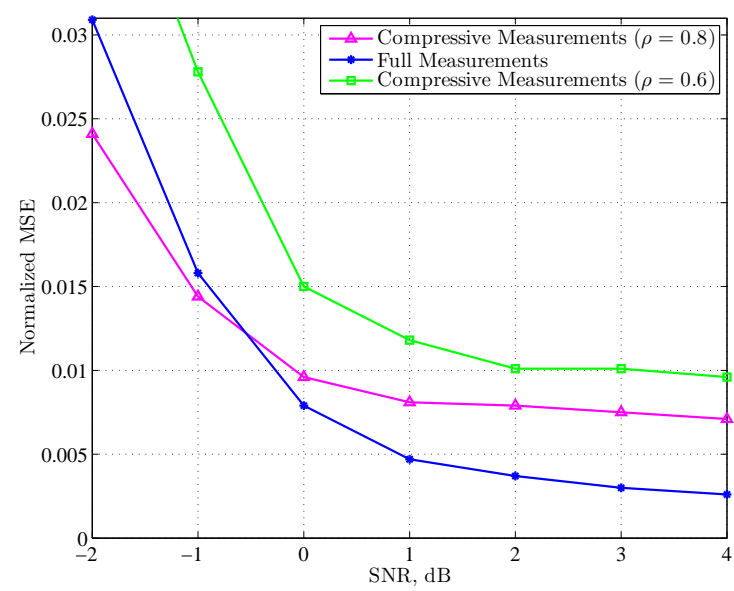

Fig. 7. Normalized Mean Square Error (MSE) versus Signal to Noise Ratio (SNR) for the correlated noise scenario (sparsity order $\sigma=0.6$, correlation coefficient $\zeta=0.6, N=100$ ) [90]. In the figure, $\rho$ denotes the compression ratio.

Measurement Vectors (MMVs). In practice, the correlated noise case may arise due to filtering and oversampling operations. Similarly, the correlated MMV case may arise due to channel correlation or imperfections in frequency selective filters present at the CR node.

Figure 7 depicts the normalized Mean Square Error (MSE) versus SNR for the correlated noise scenario for both the compressive and full measurement cases assuming correlation knowledge at the CR receiver [90], [146]. It can be deduced from the figure that the compressive case with the compression ratio $\rho=0.8$ has to sacrifice almost $0.3 \%$ estimation error in comparison to the full measurement case at $\mathrm{SNR}=1 \mathrm{~dB}$. Furthermore, this estimation error increases with the decrease in the value of $\rho$, i.e., increase in the compression. On the other hand, the advantage is that $((1-\rho) * 100) \%$ saving can be obtained in terms of hardware resources in comparison to the full measurement case. Various results on compressive SNR estimation for the correlated noise and correlated MMV cases can be found in [90].

\section{B. Compressive Sparsity Order Estimation}

For a wideband CR, sparsity order is another useful parameter to be acquired and it basically provides information about what percentage of the licensed band is available for the secondary usage. This awareness is helpful in implementing CS-based wideband sensing. Since this is a time varying parameter and is not known to the $\mathrm{CR}$ receiver as a priori, it needs to be estimated in practice. If the information about the sparsity order is available to the wideband CR transceiver, it can dynamically adapt its sampling rate in order to fully exploit the advantages of the CS technique. In this context, estimating the sparsity order is crucial and has been studied in some existing contributions [91], [127], [147].

Like in other parameter estimation problem, estimating sparsity order over a wideband requires high sampling rate, hence demanding the increased cost in the ADC hardware. In this context, it is advantageous to estimate sparsity order compressively by exploiting some sparsity basis. In this

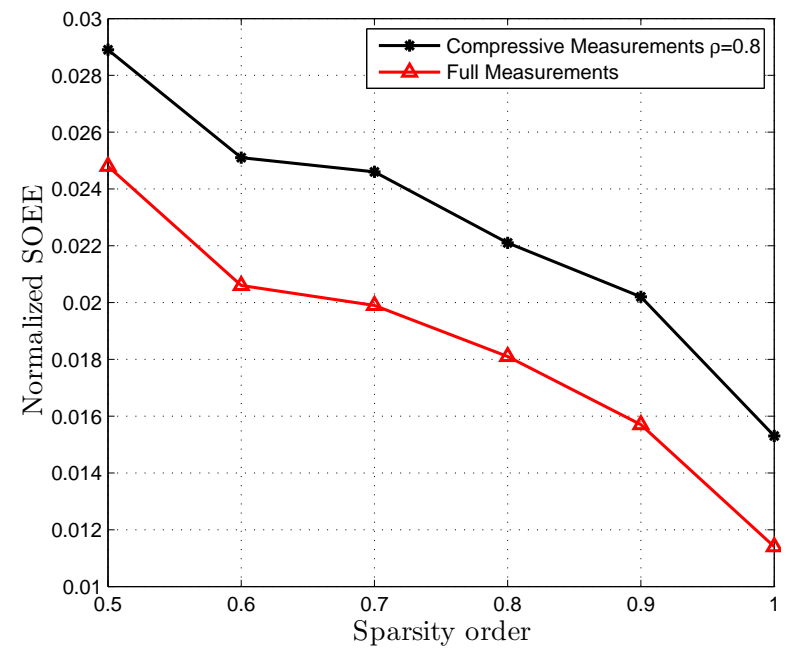

Fig. 8. Normalized Sparsity Order Estimation Error (SOEE) versus sparsity order with compressive and full measurements for the varying power scenario (Dynamic Range $\mathrm{DR}=6.02 \mathrm{~dB}, \mathrm{~N}=100$, mean power $=7.78 \mathrm{dBW}$ ) [91]. In the figure, $\rho=0.8$ denotes the $20 \%$ compression, i.e., $20 \%$ less measurements and the full measurement case indicates the Nyquist rate sampling, i.e., $\rho=1$.

context, authors in [91] have recently studied an eigenvaluebased compressive sparsity order estimation technique using asymptotic random matrix theory. The detailed theoretical analysis for the signal plus noise case has been carried out to derive the asymptotic eigenvalue probability distribution function (aepdf) of the measured signals covariance matrix for sparse signals. Subsequently, the sparsity order of the wideband spectrum has been estimated based on the derived aepdf expressions utilizing the maximum eigenvalue of the measured signal's covariance matrix.

In [91], the following three different scenarios have been considered: (i) constant received power, (ii) varying received power, and (iii) correlated scenario with the correlated MMVs. The first scenario assumes equal received power across all the carriers and uncorrelated non-zero entries across the MMVs. The second scenario allows the received power levels to vary across all the carriers vary but with a known distribution while the third scenario considers the correlated scenario in which the non-zero entries across the MMVs are correlated.

Figure 8 presents the comparison of the normalized Sparsity Order Estimation Error (SOEE) versus sparsity order for full and compressive measurement cases with parameters considering varying received power, which is modeled using a modified semicircular distribution [91], [146]. From the figure, it can be noted that sparsity order up to 0.5 can be estimated with less than $2.9 \%$ estimation error for the compressive case (with $\rho=0.8$ ) and with less than $2.5 \%$ estimation error for the full measurement case. Furthermore, it can be concluded that there exists a tradeoff between estimation performance (expressed in terms of sparsity order estimation error) and the hardware cost (number of measurements). Based on the presented results in Fig. 8, at the cost of $0.4 \%$ estimation error, $20 \%$ hardware resources can be saved since $20 \%$ less measurements are utilized. 


\section{Compressive DoA Estimation}

The DoA information is useful for CR networks or CR based sensor networks for various objectives such as adaptive beamforming, and active PU localization. For the DoA estimation problem, CS has been widely used in the literature [84][86]. Authors in [84] have studied a two dimensional (2D) bearing estimation of multiple acoustic sources with a set of sensors using a wireless channel under bandwidth constraints and the $l_{1}$-norm minimization was applied considering the target bearings as a sparse vector.

Furthermore, a CS-based architecture has been presented in [85] for array based applications by exploiting the CS in the spatial domain. The main idea behind this architecture it that a large size array can be transformed into a small size array with the random selections of the array elements. Moreover, the contribution in [86] focuses on parameter estimation using a random linear array and the CS technology. The concept behind the proposed approach in [86] is that a random linear array removes the limitation of a uniform array, and when combined with the CS reduces the burden in the design of the array.

A number of joint sparse representation methods specific to wideband DOA estimation has been studied in the literature [87], [88], [148]. Some important of these are briefly described below.

1) $l_{1}$-Singular Value Decomposition ( $l_{1}$-SVD) reconstruction: This method combines the SVD step of the subspace algorithms with a sparse recovery method based on $l_{1}$-norm minimization [148]. This algorithm can handle closely spaced correlated sources if the number of sources is known. However, it suffers from some performance degradation when the number of sources is unknown [88].

2) Joint $l_{0}$ Approximation DOA (JLZA-DOA) method: In this method, the snapshots of the measurements are represented as some jointly sparse linear combinations of the columns of an array manifold matrix and subsequently the problem is solved by using a mixed approximation approach, which is a member of the smoothed $l_{0}$ (SL0) approximation methodology [88]. These SL0 algorithms approximate the $l_{0}$-norm using a class of Gaussian functions. This algorithm can resolve the closely spaced and highly correlated sources using a small number of noisy snapshots, and does not need the prior knowledge about the number of sources.

3) Aliasing free Sparse Signal Representation (SSR) recovery method: This method is based on the the SSR-based approach which constructs steering matrices corresponding to different frequency components of the target signal [87]. The main drawback of SSR based approach is that this method is subject to ambiguity resulting from not only spatial aliasing as in classical beamforming but also from the over-completeness of the dictionary. To overcome this issue, the aliasing free SSR recovery method utilized MMVs to alleviate the ambiguity resulting from an over-complete dictionary and further uses multiple dictionaries to remove the ambiguity resulting from spatial aliasing.

\section{Compressive Channel Estimation}

In CR networks, the knowledge of the CSI towards the PRs is crucial in order to protect the PUs from the harmful interference caused by the STs while employing underlay CR techniques. Further, the channel information of the links between STs and secondary receivers is important in order to guarantee the Quality of Service (QoS) of the secondary link. Since there is no cooperation between PUs and SUs in practice, the estimation of crosslink channels is the main challenge. Moreover, the knowledge of primary channel statistics can be very helpful in making opportunistic spectrum access decisions for a CR [74]. In addition, the knowledge of the CSI information is essential for implementing advanced precoding and beamforming algorithms at the CR transmitters. Therefore, the estimation of the channel statistics efficiently and accurately is an important issue in CR networks as in legacy wireless networks. However, the conventional channel estimation methods may lead to large pilot overheads and the issue of pilot contamination in large CR networks. In this context, CS plays an important role to reduce the estimation overhead by exploiting the channel sparsity in wireless networks including the CR networks [4].

The wireless channel can often be modeled as a sparse channel in which the delay spread could be very large, but the number of significant paths is normally very small. The prior knowledge of the channel sparseness can be effectively used to improve the channel estimation using the CS theory. The common assumption used in the application of CS for channel estimation application is that a sparse multipath channel leads to a baseband channel model in which most of the channel taps are negligible [4]. The sparsity of the time domain channel can be exploited by choosing the pilots randomly. In this way, a random compressive measurement matrix can be constructed, hence conserving the available bandwidth [75].

In order to efficiently utilize the available spectral opportunities in an underlay CR network either by means of resource allocation or interference mitigation, a secondary transmitter should have the channel state information of multiple PU channels over a wide frequency band towards multiple primary receivers. In this context, CS can be helpful in reducing the estimation overhead as in other wireless networks. Furthermore, in contrast to sparse channel estimation techniques in general wireless communication channels, the pilot design in a CR network can be based on the output of spectrum sensing [76]. For example, in Orthogonal Frequency Division Multiplexing (OFDM)-based CR systems, after spectrum sensing is carried out, the OFDM subcarriers occupied by the PUs can be deactivated first, and then among the remaining active subcarriers, some subcarriers can be assigned for pilot transmission and the others to transmit data symbols for the SUs.

In the CR literature, a few works exist in the areas of sparse channel estimation using the CS approach [76]-[78]. The CS-based pilot design for sparse channel estimation in OFDM-based CR networks may help in improving the data rate and the flexibility of SUs. In this regard, authors in [76] 
studied the pilot design problem for sparse channel estimation in OFDM-based CR systems. It has been shown that the proposed spectrum sensing based sparse channel estimation can achieve $11.5 \%$ improvement in spectrum efficiency while maintaining the same performance as the traditional least square channel estimation. In the context of distributed resource allocation problem for a $\mathrm{CR}$, authors in [77] have developed a CS based estimation algorithm in order to acquire the channel and interference parameters needed for resource allocation. Furthermore, authors in [78] proposed a sparsity adaptive matching pursuit algorithm for channel estimation in Non Continuous OFDM (NC-OFDM) systems. However, the disadvantage of this sparsity adaptive matching pursuit algorithm is that it requires quite large reconstruction time. To address this issue, authors in [78] further modified the proposed algorithm as an adaptive matching algorithm and have shown that the modified adaptive matching algorithm improves the computing speed and the reconstruction accuracy as compared to those of the sparsity adaptive matching pursuit algorithm.

The compressive channel estimation has also received important attention in the area of Ultra-Wideband (UWB) technology, which can be considered as an implementation technology for the underlay CR. Due to very low Power Spectrum Density (PSD), this technology facilitates the coexistence of SUs with the PUs that operate within the UWB's wide spectrum band, i.e., 3.1 to $10.6 \mathrm{GHz}$ [79]. The transmission of ultrashort pulses in UWB technology leads to several desirable characteristics such as the rich multipath diversity introduced by a large number of propagation paths existing in a UWB channel. The rich multipath coupled with the fine time resolution of the UWB creates a challenging channel estimation problem. Fortunately, multipath wireless channels tend to exhibit impulse responses dominated by a relatively small number of clusters of significant paths, especially when operating at large bandwidths and signaling durations and/or with the numbers of antenna elements [80]. These channels are often called as "sparse channels". The conventional channel estimation methods usually provide higher errors because they ignore the prior knowledge about the sparseness of the wireless channel.

In the above context, the UWB sparse channel estimation problem has been studied in [81] under a time domain sparse model point of view. In particular, [81] defined a suitable dictionary formed by the delayed versions of the UWB transmitted pulse in order to better match the UWB signal. However, the spike basis achieves maximal incoherence with the Fourier basis and due to this reason, it seems more convenient to work with frequency domain measurements. That approach was followed in [82], where the use of CS was examined for the estimation of highly sparse channel by means of a new sparse channel estimation approach based on the frequency domain model of the UWB signal. By constructing a dictionary that closely matches the received signal (either in the time or frequency domain), the signal contributions from the strongest paths of the UWB multipath channel can be recovered from the set of random projections of the received pilot signals.

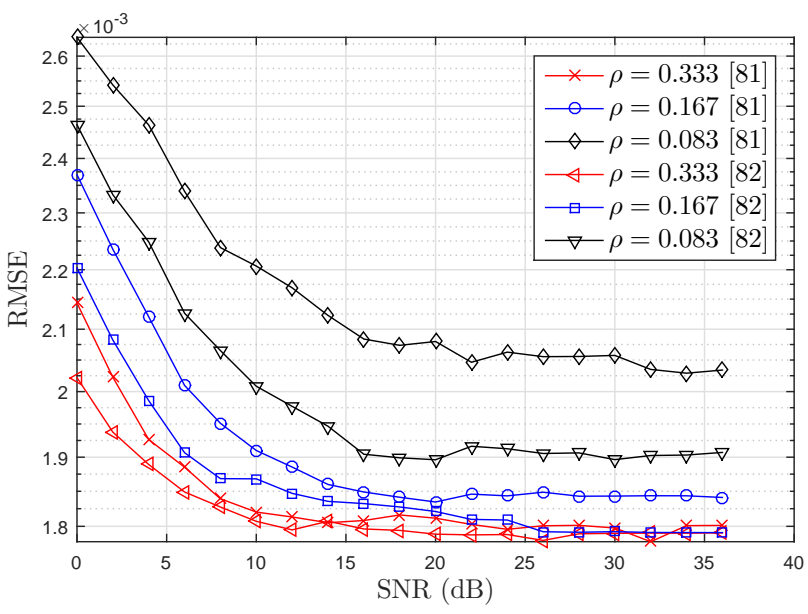

Fig. 9. Comparison of the CS-based channel estimation techniques in [81] and [82] in terms of RMSE of the reconstructed channel versus SNR.

Figure 9 depicts the Root Mean Square Error (RMSE) of the recovered signal for both models ( [81] and [82]). It can be observed that when the compression rate $\rho$ is high, both perform equally well but, as the compression rate decreases, the error obtained with [81] increases faster than the error obtained with [82] as we move to lower SNR region.

\section{Compressive Radio EnVIRONMENT MaP (REM) CONSTRUCTION}

In this section, first, we highlight the importance of the Radio Environment Map (REM) construction utilizing the CS approach, i.e., compressive REM construction, for the implementation of CR networks. Subsequently, we review the existing related works in the context of compressive REM construction highlighting the challenges in heterogeneous environment. Then we propose a generalized framework for constructing the REM in REM settings along with the main issues to be addressed.

\section{A. Importance in CR Networks}

The REM is an architectural concept for storing environmental information for use in CR networks [149]. This facilitates the geolocation database-assisted CR communications which is an alternative spectrum awareness mechanism to obtain the knowledge of the RF environment. One way of constructing the REM is to use the spectrum cartography method which is the process of plotting an attribute of the RF environment over a finite geographical area. Spectrum cartography has important applications in network planning, maintenance and optimization, and has been widely used by the cellular network designers. In CR applications, spectrum cartography can be used as a powerful tool to determine the presence and the range of active PU transmitters [96], [99].

For the effective implementation of CR networks, an REM can be extremely useful for the proper selection of the SU channel and the transmit parameter. The accurate selection of these parameters must be made considering the requirements of PU interference control and the QoS of the secondary 
link, of which the first requirement is the more crucial. This interference control can only be guaranteed only if the PU locations and the received powers levels from other PUs are known by the SUs. Therefore, the locations of the transmitters and their transmit power levels need to be accurately estimated in order to construct an REM. Subsequently, the map of the received power level throughout a two dimensional area can be created utilizing the estimated locations and the transmit power levels.

In practice, it is highly desirable to construct the REM over a wide coverage area and over a wide spectrum band. However, this requires a large number of sensors and a high overhead on parameter acquisition and recovery while applying the conventional non-CS based approaches. In this context, the following two practical aspects motivate the use of the CS in REM construction problems.

1) As mentioned earlier, there is a small number of active carriers compared to the total number of carriers used in the legacy systems, thus creating the sparsity in the frequency domain.

2) There is a small number of active primary transmitters compared to the total number of distributed sensors, thus creating the sparsity in the spatial domain.

By utilizing the above sparsity bases, system designers can take advantage of the CS method in constructing the REM over a wideband area and a wideband spectrum band.

\section{B. Related Literature}

There exist several spectrum cartography works in the literature [150]-[152], which do not exploit the sparsity of the active PUs in space and frequency domains. In most of these works, spatial interpolation, which is commonly used in Geographic Information Systems (GIS), has been used. It refers to any system manipulating geographical referenced data for capture, storage, analysis and management purposes. The main spatial interpolation techniques are the Inverse Distance Weighting (IDW), the Nearest Neighbor Interpolation, Splines and Kriging [153].

Determining the location and power level of the active PTs considering the sparsity feature of the primary activity significantly helps in constructing an REM. In this regard, several researchers have exploited this sparsity in various settings [92], [96], [97], [154]. One of the widely used CS-based REM construction method relies on a location fingerprinting approach [92], [96], [97]. In this approach, PUs and SUs are assumed to be located in a random subset of the grid points within a certain discretized geographic area. The RSS from the target PUs is measured by each SU and this set of measurements is used to recover the PU locations and transmit power levels. In many cases, the sparsity is exploited assuming that the number of active PUs is much smaller than the number of grid points.

In a sparse target localization problem, the main objective is to determine the locations of the targets, i.e., active transmitters, simultaneously using a relatively small number of noisy Received Signal Strength (RSS) measurements. In this context, authors in [92] present a framework for localizing multiple

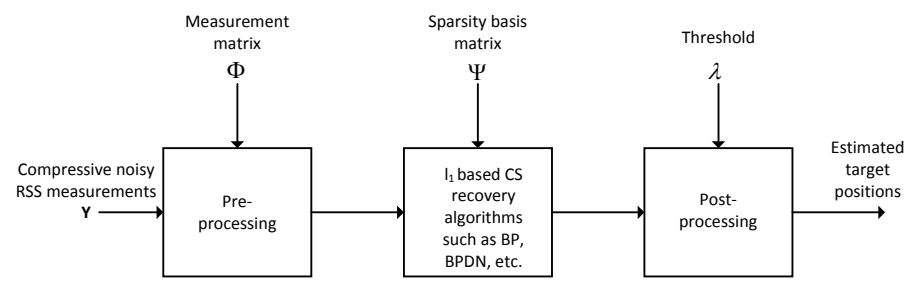

Fig. 10. The flow chart of CS-based multiple target localization approach [92]

targets/transmitters utilizing the CS theory. The multiple target localization problem has been formulated as a sparse matrix in the discrete spatial domain and an RSS-based algorithm has been used to find the location of targets. The flow chart of the CS-based multiple target localization approach studied in [92] is depicted in Fig. 10 and the involved steps are briefly summarized in the following paragraph.

The $M \times K$ input matrix $\mathbf{Y}$ in Fig. 10 consists of compressive noisy RSS measurements taken by $K$ targets on $M$ arbitrary reference points. For the considered target localization problem in [92], the sparsity basis $\Psi$ and the measurement matrix $\boldsymbol{\Phi}$ are coherent in the spatial domain and CS theory can not be directly applied. In this context, one approach to apply the CS principle is to carry out pre-processing operation on the measured matrix $\mathbf{Y}$, which results in the same effect as in orthogonalizing two matrices. After preprocessing, the original sparse coefficients can be recovered from the compressive noisy measurements using suitable $l_{1}$ minimization algorithms such as BP, Basis Pursuit Denoising (BPDN), etc. Then, post-processing operation is required in order to compensate for the grid assumption error since the targets may not be exactly located at these grid points. For this purpose, the dominant coefficients, whose values are above a certain threshold $\lambda$, can be found and then the centroid of these grid points, which acts as the location indicator, can be calculated as illustrated in [92].

Besides the application of CS in spectrum cartography and target counting/localization, $\mathrm{CS}$ is relevant when creating interference maps in various wireless networks such as IEEE 802.22 Wireless Regional Area Networks (WRAN), which is the first CR-based wireless standard. In a typical cellular network like WRAN, user terminals have to communicate via the base station and they cannot communicate with each other directly. To address this drawback and to take advantage of both centralized control and the sharing of spectrum among user terminals, Peer to Peer WRAN (P2PWRAN), which requires the information regarding potential interference among flows, i.e., interference map, has been proposed in [93]. The accuracy of this interference map affects the channel allocation as well as the network performance.

In the above context, authors in [94] have proposed selfadapting interference mapping protocol in order to cope with the unexpected events in P2PWRAN networks without considering CS aspects. In practice, it's not realistic to capture the global information about the spectrum usage in its surrounding environment using a single $\mathrm{CR}$ transmitter. The problem of reconstructing the spectrum map using incomplete information 
becomes interesting since a CR transmitter can acquire only the local data from a limited number of cooperative nodes. Due to ability of the CS technique to sample and compress simultaneously, it can serve the requirement of fast sensing in a CR. Furthermore, a spectrum map facilitates the definition of the QoS constraint based on Signal to Interference plus Noise Ratio (SINR), thus enabling the simultaneous operation of the routing and cooperative spectrum sensing [95]. Subsequently, it is applicable in creating interference maps with the help of CR sensors. In contrast to the statistical power spectral density map creation for spatial frequency reuse in [154], authors in [95] have proposed aggregated interference-based deterministic power-level maps for routing using significantly less measurements compared with the traditional non-CS methods.

Furthermore, authors in [154] proposed a Least Absolute Shrinkage and Selection Operator (LASSO) based distributed algorithm which exploits sparsity to construct PU Power Spectral Density (PSD) and to reveal the unknown positions of the active PUs. The following two forms of sparsity are used in [154]: (i) the sparsity introduced by the narrow-band nature of transmit-PSDs relative to the broad range of the usable spectrum, and (ii) the sparsity emerging from sparsely located active radios in the operational space. It has been demonstrated that exploiting sparsity in the distributed CR sensing reduces spatial and frequency spectrum leakage by $15 \mathrm{~dB}$ relative to the least square alternatives.

In the similar context, authors in [96] propose an OMP algorithm-based technique [155] considering the cartography process as a CS problem. The proposed Orthogonal Matching Pursuit Spatial Extension (OMPSE) algorithm exploits the spatial correlation between two nearby reference points in a neighbourhood and provides better performance over the traditional OMP technique. Additionally, the authors in [99] investigated the performance of CS-based cartography process in a fading environment where real time channel estimation may not be available. The well-known iteratively reweighted $l_{1}$ minimization approach has been extended by exploiting spatial correlation between two points in space in order to accommodate the lack of channel information.

In practice, Wireless Sensor Networks (WSNs) can be used to acquire the information about the surrounding radio environment in order to construct an REM. In WSNs, the following two problems can be addressed by the CS approach [156]. First, there are a very limited number of active sensors, i.e., the active PU transmitters, compared with the total number of sensors in the network. Moreover, the number of events is much less compared to the number of all sources. Second, different events may happen simultaneously and cause interference to detect them individually. As a result, the received signals are superimposed all together, and an efficient algorithm is required to separate the superimposed signals.

Besides, it's highly costly to deploy a WSN over a large geographical area solely for the purpose of constructing the REM. To address this issue, the network operators can opportunistically use the heterogeneous RF devices in the desired area that are deployed for functions other than spectrum mapping. In this context, the spectrum cartography system has to account for different radio types, propagation environments, and sensor densities as well as sparse receiver measurements. Further, the system must be scaled based on the number of users that must be supported [98].

In a practical heterogeneous environment which may consist of heterogeneous sensor devices, there exist the following challenges for creating a reliable dynamic spectrum mapping system [98].

- Dedicated resources may not be available for the sensing tasks and we may need to rely on the opportunistic use of the devices deployed for other purposes. Besides, due to several structural, operational, and economic practical constraints, we have to rely on the sparse measurements.

- The spectrum awareness has to be carried out over a wide frequency band that may be heavily occupied with a large number of heterogeneous sources/PU transmitter types. Although current methods utilize multiple, identical, broadband, high performance spectrum analyzers in the vicinity of the sensors, this solution is neither costeffective nor easily scalable.

- The WSNs deployed for the purpose of gathering the RF information should operate in a dynamic RF environment with diverse propagation constrained network infrastructure with the minimal impact on the main objective of the sensors. Further, as the number of devices and the rate of data collection increase, scalable solutions are needed to store, process, retrieve, and disseminate the information.

- The sensor network may consist of heterogeneous RF devices over a large geographical area. The coordination between these heterogeneous devices is highly desirable from the practical perspective.

In the context of heterogeneous environment, authors in [98] have studied a method, called PRISM (Precise RadioPropagation Interpolation from Sparse Measurements). This method takes the sparse spatial measurements and uses sparse signal reconstruction techniques in order to determine the simplest arrangement of virtual sources consistent with the observations. Furthermore, Kanerva's sparse distributed memory model has been used to address system scaling challenges which represents the $N$-dimensional binary vectors without regard to the semantic interpretation of the data.

Moreover, target counting and localization are the key aspects in order to construct the REM using the sensor networks. Although there exists much literature in the field of non-CS based target counting, only a few recent contributions have focussed on CS-based target counting and localization [97], [156], [157], [159]-[164]. Among these contributions, [156], [157] have focussed on CS-based target counting, [97], [159] have focussed on both target counting and localization, and the rest have focussed CS-based localization. The contribution in [157] proposed a CS-based approach for sparse target counting and positioning in wireless sensor networks by employing a novel Greedy Matching pursuit (GMP) algorithm. Recently, authors in [158] studied the problem of target counting and localization by exploiting the joint sparsity feature of an MMV model and demonstrated that the performance of the proposed MMV approach is superior than that of the conventional single 
measurement vector method in terms of target counting and localization accuracies.

\section{Framework for Compressive REM Construction and Re- lated Issues}

As stated earlier, the main objectives of compressive REM construction over a wideband coverage area are

- To recover the active carriers within the area of interest

- To create an REM for each active carrier based on compressive measurements

Thus constructed REM can be used to implement carrier assignment and power control for secondary devices of the CR networks.

Let us consider a grid of $L$ sensors spanning a coverage area of interest. Within the same area, there is usually an unknown small number of active Txs, i.e., $K<<L$. In general, the following parameters have to be recovered to reconstruct the REM: (i) number of active Txs $K$, (ii) position of Txs, and (iii) radiated power for each Tx. The Txs are assumed to be collocated with one of the sensors. If this is not the case, appropriate interpolation techniques can be employed. In a centralized setting, the sensors measure the received energy over a number of samples and relay these measurements to the FC. The reporting links from the sensors to the FC are usually bandwidth limited and need to be utilized effectively. In this context, it can be assumed that the received measurements are relayed to the $\mathrm{FC}$ through $C$ number of wireless collectors. Further, due to the limitation in the backhaul link bandwidth, only $D$ out of $C$ wireless collectors can be accessed simultaneously at each time slot. It can be assumed that this access pattern is randomly generated.

Let $x_{i}, i=1 \ldots L$ denote the locations of sensors, while $y_{i}, i=1 \ldots K$ the locations of active transmitters and $p_{i}, i=$ $1 \ldots K$ the transmit power of the PU Txs. In vectorial form, we can define the $L \times 1$ vector $\mathbf{x}$ and the sparse $K \times 1$ vector $\mathbf{s}=\mathbf{y} \odot \mathbf{p}$. To construct the compressive REM in the above setting, the objective is to estimate the following parameters: (i) the number of non-zero points (sparsity order) $K$, (b) the positions of non-zero points $y_{i}$, and (ii) the values of non-zero points $p_{i}$

Assuming that the sensor locations are known through GPS feedback, the following relation can be applied

$$
\mathbf{x}=\mathbf{A s},
$$

where $\mathbf{A}$ is a $K \times K$ channel matrix including the transmit, receive antenna gains and the path loss calculated based on distances between sensors. This can also be target energy decay matrix as considered in [157]. Similarly, assuming that the locations of the collectors is known, the output at the active collectors can be expressed as a $D \times 1$ vector $\mathbf{w}$, given by

$$
\mathbf{w}=\mathbf{B} \mathbf{x}=\mathbf{B A s}+\mathbf{z},
$$

where $\mathbf{B}$ is a $D \times K$ power law path loss matrix calculated based on the distances between the collector and sensors, and $\mathbf{z}$ is additive white Gaussian noise vector. Since the selection of active collectors is random, the matrix $\mathbf{B}$ is also random with positive elements. Finally, at the FC, arbitrary CS matrices such as $\mathbf{C}$ can be applied in such a way that the received sample vector $\mathbf{v}$ becomes

$$
\mathbf{v}=\mathbf{C w}
$$

Based on the above formulation, we highlight the main issues to be considered for future investigation below.

- How to estimate the parameters without completely reconstructing the signal and with lower complexity?

- How to design B in order to satisfy the incoherency with A and the RIP property?

- How to design $\mathbf{C}$ which is practically implementable?

- Are multiple sample vectors $\mathbf{v}$ helpful to improve performance in the above setting?

\section{Challenges and Future Recommendations}

In this section, we highlight the main challenges and suggest future recommendations to address them.

\section{A. Alleviating Existing Assumptions}

- Requirement of Channel Occupancy Knowledge: In most of the contributions using the CS approach, it is assumed that the sparsity of a signal to be acquired is known. However, in the context of CR networks, the prior information about the PU channel occupancy may not been known to the CR sensor. This creates a great barrier to the practical usage of CS in CR scenarios. The possible way forward for this would be to investigate suitable sparsity order estimation method in order to estimate the sparsity order of the wideband spectrum accurately [91]. Further, the sparsity order of the wideband signal generally varies over the time and it may be difficult to estimate it in practice due to the dynamicity of the primary spectrum usage or the time-varying fading channels between CRs and the PUs [104]. Moreover, the required sampling rate changes proportionally with the sparsity order of the wideband signal. Therefore, it's an important research challenge to investigate adaptive sparsity order estimation methods to capture the dynamicity of the spectrum usage in time-varying wireless environments, and subsequently to choose an appropriate sampling rate to be applied at the CS-based CR transceiver.

- Investigation of Suitable Sparsity Basis: On the one hand, most existing CS works in the context of CR communications assume the sparsity of the radio spectrum in the frequency domain due to its lower utilization. On the other hand, the main objective of CR communications is to enhance the usage of the spectrum. In this regard, the consideration of sparsity in the frequency domain may be relevant for the current scenario but the situation may change in the future due to the enhanced usage of the radio spectrum [166]. Therefore, it's crucial to investigate suitable sparsity bases/domains in order to apply CS in $C R$ applications. One solution would be to exploit the sparsity in different features of the primary signals such as spectral correlation function which is sparse in both cyclic and angular frequency domains. 
- Basis Mismatch in Compressive Estimation/Detection: In many CS problems, the field/signal to be acquired is assumed to be sparse in some basis. However, in practice, the signal may not be sparse in any a priori known basis, resulting in the basis mismatch [44]. In this context, it is important to analyze the sensitivity of CS to mismatch between the assumed and the actual sparsity bases and to investigate suitable approaches to reduce this mismatch. Moreover, developing robust CS approaches with unknown basis is an important research challenge.

- Evaluation under Realistic Signal Model: In most of the existing contributions, a random signal model is considered in numerical experiments. However, it does not provide an adequate description of real signals, whose frequencies and phases significantly differ from that of the random signal [59]. In this context, it is crucial to investigate the performance of CS algorithms for real signals in the considered CR scenarios. Furthermore, in most of the CR related CS research, finite-length and discrete-time signals have been considered [104]. In this context, investigating low-complex solutions to implement the CS-based signal processing techniques in the analog domain should be one of the focus areas in the future research.

\section{B. Tackling Implementation Aspects}

- Design of a Practical Measurement Matrix: Another issue in designing practical CS-based receivers is to investigate suitable practical sensing matrices. The compression of a non-sparse signal depends on the proper selection of the measurement matrix. Furthermore, the costs for CS encoding and decoding significantly depends on the type of measurement matrix [50]. The well-known family of CS matrices is a random projection or a matrix of i.i.d. random variables from a sub-Gaussian distribution such as Gaussian or Bernoulli since this family is universally incoherent with all other sparsifying bases [165]. However, in practical applications, the unstructured nature of random matrices make their realizations highly complex and the memory requirement also increases. In this context, it is an open challenge to investigate practical sensing matrices required for compressive detection/estimation applications.

- Compressive REM Construction in Heterogeneous Networks: REMs are useful for the implementation of database-based cognitive communication over a wide coverage area and wide bandwidth. They can be created with the help of distributed sensor measurements. However, due to the constraints on the sensor hardware and energy, it is necessary to keep the number of sensor measurements low. As highlighted in Section V, there exist several practical issues while constructing REM with the help of compressive measurements. Some of the important issues are diverse propagation characteristics, no or imperfect knowledge about the primary network, practical constraints on the sensor placement, constrained network infrastructure, scalability with the future sensor nodes etc. Furthermore, the issues mentioned in Section $\mathrm{V}-\mathrm{C}$ should be considered in the future research towards implementing the compressive REM construction. In this context, the application of CS for REM construction by exploiting the sparsity in the frequency and spatial domains can be considered as an interesting future research problem.

Furthermore, in the spectral coexistence scenario of satellite and terrestrial networks [11], elevation angle provides an additional degree of freedom in enabling this coexistence [167]. However, the existing REM construction methods do not take into account of the elevation angle. In this context, it's an important future topic to explore a suitable construction method for three dimensional (3D) REM considering elevation angle as an additional dimension.

\section{Performance Limits under Practical Imperfections}

Most existing CS techniques assume system models contaminated with either Guassian noise with the known variance or the bounded noise. Furthermore, most CS-based works in the context of CR communications assume ideal operating conditions in terms of noise, channel and transceiver hardware components and there exist only a few works investigating CS-based techniques in the presence of practical imperfections such as interference [168], and noise uncertainty [169]. However, in practice, there may occur various imperfections such as noise uncertainty, channel uncertainty, noise/channel correlation, and transceiver hardware imperfections such as ADC errors (quantization and clipping errors), synchronization errors, amplifier non-linearity, multicarrier distortion, calibration etc [22]. For example, the centralized compressive collaborative approach (Fig. 4) studied in [64], [129] considers ideal reporting channels, which is not the case in practice. These imperfections may lead to significant performance degradation of a CS-based techniques in practical CR communications. Therefore, it's an important future step to investigate the practical gains that can be achieved with CS-based approaches in the presence of practical imperfections and to develop a common framework to combat their aggregate effect in a CSbased CR transceiver.

\section{CONCLUSIONS}

CS has been well motivated for CR communications due to the sparse nature of the radio spectrum occupancy in practical wireless systems. In this context, this paper has provided a comprehensive review on the applications of CS in CR communications. Starting with the basic principles and the main aspects of the CS technique, this paper has identified various application areas such as wideband SS, environmental parameter estimation and REM construction based on the RF parameter to be acquired. Subsequently, dynamic range and sampling rate issues for wideband SS have been discussed and the existing related works have been reviewed. Furthermore, the existing works on compressive estimation of various parameters such as SNR, sparsity order, DoA and 
channel have been detailed in the context of a CR. Moreover, the state of the art approaches on the compressive REM construction have been discussed and a generalized framework has been presented. Finally, some open issues which need to be considered in the future research have been identified.

\section{REFERENCES}

[1] E. J. Candes and M. Wakin, "An introduction to compressive sampling," IEEE Signal Process. Mag., vol. 25, no. 2, 2008, pp. 21-30.

[2] E. J. Candes, "Compressive sampling," in Proc. Int. Congress of Mathematicians, vol. 3, 2006, pp. 1433-1452.

[3] M. F. Duarte, et al., "Single-Pixel Imaging via Compressive Sampling," IEEE Signal Process. Mag., vol. 25, no. 2, pp. 83-91, Mar. 2008.

[4] C. Berger, Z. Wang, J. Huang, and S. Zhou, "Application of compressive sensing to sparse channel estimation," IEEE Commun. Mag., vol. 48, no. 11, pp. $164-174$, Nov. 2010.

[5] Y. Peng, X. Yang, X. Zhang, W. Wang, and B. Wu, "Compressed MIMO-OFDM channel estimation," in Proc. 12th IEEE Int. Conf. on Commun. Technol. (ICCT), Nov. 2010, pp. $1291-1294$.

[6] R. Baraniuk and P. Steeghs, "Compressive radar imaging," in Proc. IEEE Radar Conf., Apr. 2007, pp. 128 -133.

[7] Z. Tian and G. Giannakis, "Compressed sensing for wideband cognitive radios," in Proc. IEEE Int. Conf. Acoustics, Speech and Signal Process., Apr. 2007, pp. $1357-1360$.

[8] A. Massa, P. Rocca, and G. Oliveri, "Compressive Sensing in Electromagnetics - A Review,", IEEE Antennas and Propag. Mag., vol. 57, no. 1, pp. 224-238, Feb. 2015.

[9] J. Mitola and G. Q. Maguire, "Cognitive radio: making software radios more personal," IEEE Personal Commun., vol. 6, no. 4, pp. $13-18$, Aug. 1999.

[10] C.-S. Sum, et al., "Cognitive communication in TV white spaces: An overview of regulations, standards, and technology," IEEE Commun. Mag., vol. 51, no. 7, pp. 138-145, July 2013.

[11] S. K. Sharma, S. Chatzinotas, and B. Ottersten, "Cognitive radio techniques for satellite communication systems," in Proc. IEEE Veh. Technol. Conf. (VTC Fall), Sept. 2013, pp. 1-5.

[12] S. K. Sharma, S. Chatzinotas, and B. Ottersten, "Satellite cognitive communications: Interference modeling and techniques selection," in Proc. 6th ASMS and 12th SPSC, Sept. 2012, pp. 111-118.

[13] J. Romberg and M. Wakin, "Compressed sensing: A tutorial," in Proc. IEEE Statistical Signal Process. Workshop, Wisconsin, 2007.

[14] M. Alodeh, S. Chatzinotas, and B. Ottersten, "Spatial DCT-Based Channel Estimation in Multi-Antenna Multi-Cell Interference Channels," IEEE Trans. Signal Process., vol. 63, no. 6, pp. 1404-1418, Mar. 2015.

[15] S. K. Sharma, M. Patwary, and M. Abdel-Maguid, "Spectral efficient compressive transmission framework for wireless communication systems," IET Signal Process., vol. 7, no. 7, pp. 558-564, Sept. 2013.

[16] T. Yucek and H. Arslan, "A survey of spectrum sensing algorithms for cognitive radio applications," IEEE Commun. Surveys \& Tutorials, vol. 11 , no. 1 , pp. $116-130,2009$.

[17] K. Patil, R. Prasad, and K. Skouby, "A survey of worldwide spectrum occupancy measurement campaigns for cognitive radio," in 2011 Int. Conf. Devices and Communications (ICDeCom), Feb. 2011, pp. 1-5.

[18] I. Akyildiz, W.-Y. Lee, M. C. Vuran, and S. Mohanty, "A survey on spectrum management in cognitive radio networks," IEEE Commun. Mag., vol. 46, no. 4, pp. 40-48, Apr. 2008.

[19] J. Wang, M. Ghosh, and K. Challapali, "Emerging cognitive radio applications: A survey," IEEE Commun. Mag., vol. 49, no. 3, pp. 7481, Mar. 2011.

[20] M. Masonta, M. Mzyece, and N. Ntlatlapa, "Spectrum decision in cognitive radio networks: A survey," IEEE Commun. Surveys \& Tutorials, vol. 15, no. 3, pp. 1088-1107, 2013.

[21] A. De Domenico, E. Strinati, and M. Di Benedetto, "A survey on MAC strategies for cognitive radio networks," IEEE Commun. Surveys \& Tutorials, vol. 14, no. 1, pp. 21-44, 2012.

[22] S. K. Sharma, T. Bogale, S. Chatzinotas, B. Ottersten, L. Le, and X. Wang, "Cognitive Radio Techniques under Practical Imperfections: A Survey," IEEE Commun. Surveys \& Tutorials, vol. 17, no. 4, pp. 18581884, Fourth quarter 2015.

[23] B. Wang and K. Liu, "Advances in cognitive radio networks: A survey," IEEE J. Sel. Topics in Signal Process., vol. 5, no. 1, pp. 5-23, Feb. 2011.
[24] R. Baraniuk, "Compressive sensing [lecture notes]," IEEE Signal Process. Mag., vol. 24, no. 4, pp. 118 -121, July 2007.

[25] D. Donoho, "Compressed sensing," IEEE Trans. Info. Th., vol. 52, no. 4, pp. $1289-1306$, Apr. 2006.

[26] M. Aharon, M. Elad, and A. Bruckstein, "K-SVD: An Algorithm for Designing Overcomplete Dictionaries for Sparse Representation," IEEE Trans. Signal Process., vol. 54, no. 11, pp. 4311-4322, Nov. 2006.

[27] S. S. Chen, D. L. Donoho, and M. A. Saunders, "Atomic Decomposition by Basis Pursuit," SIAM J. Scientific Computing, vol. 20, no. 1, pp. 33-61, 1999.

[28] S. Boyd and L. Vandenberghe, Convex Optimization. Cambridge University Press, 2004.

[29] E. J. Candes, J. Romberg, and T. Tao, "Robust Uncertainty Principles: Exact Signal Reconstruction From Highly Incomplete Frequency Information," IEEE Trans. Info. Th., vol. 52, no. 2, pp. 489-509, 2006.

[30] E. J. Candes and T. Tao, "Near Optimal Signal Recovery from Random Projections: Universal Encoding Strategies," IEEE Trans. Info. Th., vol. 52, no. 12, pp. 5406-5425, Dec. 2006.

[31] S. G. Mallat and Z. Zhang, "Matching Pursuits with Time-Frequency Dictionaries," IEEE Trans. Signal Process., vol. 41, no. 12, pp. 33973415, Dec. 1993.

[32] J. A. Tropp, "Greed is Good: Algorithmic Results for Sparse Approximation," IEEE Trans. Info. Th., vol. 50, no. 10, pp. 2231-2242, Oct. 2004.

[33] M. Elad, "Sparse and Redundant Representations: From Theory to Applications in Signal and Image Processing”, Springer, 2010.

[34] D. Ramasamy, S. Venkateswaran, and U. Madhow, "Compressive parameter estimation in AWGN," IEEE Trans. Signal Process., vol. 62, no. 8, pp. 2012-2027, 2014

[35] M. Wakin, "Manifold-based signal recovery and parameter estimation from compressive measurements," 2008, Available: http://arxiv.org/abs/1002.1247.

[36] J. Haupt, R. Castro, R. Nowak, G. Fudge, and A. Yeh, "Compressive sampling for signal classification,” Proc. Asilomar Conf. Signals, Syst. Comput., Pacific Grove, CA, USA, Oct. 2006.

[37] Zhi Tian, E. Blasch, Wenhua Li, Genshe Chen, and Xiaokun Li, "Performance evaluation of distributed compressed wideband sensing for cognitive radio networks," in 11th International Conference on Information Fusion, pp.1-8, June 30-July 32008.

[38] B. L. Sturm, and M. G. Christensen, "Comparison of orthogonal matching pursuit implementations," in Proc. 20th European Signal Process. Conf. (EUSIPCO), pp. 220-224, Aug. 2012.

[39] Z. Qin, Y. Gao, and C. G. Parini, "Data-Assisted Low Complexity Compressive Spectrum Sensing on Real-Time Signals under SubNyquist Rate," in IEEE Transactions on Wireless Communications, vol. PP, no. 99, pp.1-1, doi: 10.1109/TWC.2015.2485992.

[40] D. Wipf, and S. Nagarajan, "Iterative Reweighted $l_{1}$ and $l_{2}$ Methods for Finding Sparse Solutions," in IEEE J. Sel. Topics Signal Process., vol. 4, no. 2, pp. 317-329, April 2010.

[41] S. Hong, "Direct spectrum sensing from compressed measurements," in IEEE Military Commun. Conf., pp. 1187-1192, Oct. 31-Nov. 3, 2010.

[42] M. Davenport, P. Boufounos, M. Wakin, and R. Baraniuk, "Signal processing with compressive measurements," IEEE J. Sel. Topics in Signal Process., vol. 4, no. 2, pp. 445-460, 2010.

[43] M. Duarte, M. A. Davenport, M. Wakin, and R. G. Baraniuk, "Sparse signal detection from incoherent projections," in IEEE ICASSP, May 2006.

[44] Y. Chi, L. Scharf, A. Pezeshki, and A. Calderbank, "Sensitivity to basis mismatch in compressed sensing," in IEEE Trans. Signal Process., vol. 59, no. 5, May 2011, pp. 2182-2195.

[45] G. Tang, B. Bhaskar, P. Shah, and B. Recht, "Compressed sensing off the grid," IEEE Trans. Info. Th., vol. 59, no. 11, pp. 7465-7490, Nov. 2013.

[46] T. Wimalajeewa, H. Chen, and P. Varshney, "Performance limits of compressive sensing-based signal classification," in IEEE Trans. Signal Process., vol. 60, no. 6, June 2012, pp. 2758-2770.

[47] M. Davenport, M. Duarte, M. Wakin, J. Laska, D. Takhar, K. Kelly, and R. Baraniuk, "The smashed filter for compressive classification and target recognition," in Proc. SPIE, San Jose, CA, Jan. 2007.

[48] A. Goldsmith, S. Jafar, I. Maric, and S. Srinivasa, "Breaking spectrum gridlock with cognitive radios: An information theoretic perspective," Proc. IEEE, vol. 97, no. 5, pp. 894 -914, May 2009.

[49] M. Davenport, J. Laska, J. Treichler, and R. Baraniuk, "The pros and cons of compressive sensing for wideband signal acquisition: Noise folding versus dynamic range," IEEE Trans. Signal Process., vol. 60, no. 9 , pp. $4628-4642$, Sept. 2012. 
[50] J. Tropp, M. Wakin, M. Duarte, D. Baron, and R. Baraniuk, "Random filters for compressive sampling and reconstruction," in IEEE Int. Conf. Acoustics, Speech and Signal Process., vol. 3, May 2006.

[51] J. Zhang, Y. Ban, D. Zhu and G. Zhang, "Random filtering structurebased compressive sensing radar", EURASIP J. Advances in Signal Process., vol. 94, 2014.

[52] Y. Wang, Z. Tian, and C. Feng, "A two-step compressed spectrum sensing scheme for wideband cognitive radios," in IEEE Global Telecommun. Conf., Dec. 2010, pp. 1 -5.

[53] J. P. Slavinsky, J. N. Laska, M. A. Davenport, and R. G. Baraniuk, "The compressive multiplexer for multi-channel compressive sensing," in IEEE Acoustics, Speech and Signal Processing (ICASSP), May 2011, pp. $3980-3983$.

[54] A. Ahmed, and J. Romberg, "Compressive Multiplexing of Correlated Signals," IEEE Trans. Info. Th., vol. 61, no. 1, pp. 479-498, Jan. 2015.

[55] E. Lagunas, "Compressive Sensing Based Candidate Detector and its Applications to Spectrum Sensing and Through-the-Wall Radar Imaging", PhD Thesis, Polytechnique University of Catalonia (UPC), Barcelona, Spain, Mar. 2014.

[56] H. Urkowitz, Harry, "Energy detection of unknown deterministic signals", Proceedings of the IEEE, vol. 55, no. 4, pp. 523-531, April 1967.

[57] A. V. Dandawate and G. B. Giannakis, "Statistical tests for presence of cyclostationarity," IEEE Trans. Signal Process, vol. 42, no. 9, pp. 23552369 , Sep. 1994.

[58] A. Jung, Z. Ben-Haim, F. Hlawatsch, and Y. C. Eldar, "Unbiased Estimation of a Sparse Vector in White Gaussian Noise," IEEE Transactions on Information Theory, vol. 57, no. 12, pp. 7856-7876, Dec. 2011.

[59] J. Tropp, J. Laska, M. Duarte, J. Romberg, and R. Baraniuk, "Beyond Nyquist: Efficient sampling of sparse bandlimited signals," IEEE Trans. Info. Th., vol. 56, no. 1, pp. $520-544$, Jan. 2010.

[60] N. Li, and H. Qian, "The performance of multichannel random demodulator for multiband signals," Proc. IEEE Int. Conf. Signal Process., pp. 1-5, Aug. 2013.

[61] Yilun Chen, M. Mishali, Y. C. Eldar, and A. O. Hero, "Modulated wideband converter with non-ideal lowpass filters," Proc. IEEE Int. Conf. Acoustics Speech and Signal Processing (ICASSP), pp. 36303633, Mar. 2010

[62] A. Harms, W. U. Bajwa, and R. Calderbank, "A Constrained Random Demodulator for Sub-Nyquist Sampling," IEEE Trans. Signal Process., vol. 61, no. 3, pp. 707-723, Feb. 2013.

[63] M. Mishali and Y. Eldar, "From theory to practice: Sub-Nyquist sampling of sparse wideband analog signals," IEEE J. Sel. Topics in Signal Process., vol. 4, no. 2, pp. 375 -391, Apr. 2010.

[64] J. Meng, W. Yin, H. Li, E. Hossain, and Z. Han, "Collaborative spectrum sensing from sparse observations in cognitive radio networks," IEEE J. Sel. Areas in Commun., vol. 29, no. 2, pp. 327 -337, Feb. 2011.

[65] Z. Fanzi, C. Li, and Z. Tian, "Distributed compressive spectrum sensing in cooperative multihop cognitive networks," IEEE Sel. Topics in Signal Process., vol. 5, no. 1, pp. 37-48, Feb. 2011.

[66] S. Gao, K. Li, L. Gan, and C. Ling, "Wyner-ziv coding for distributed compressive sensing," Sensor Signal Process. for Defence (SSPD), pp. $1-5,2011$.

[67] A. Elzanati, M. Abdelkader, K. Seddik, and A. Ghuniem, "Collaborative compressive spectrum sensing using Kronecker sparsifying basis," in IEEE Wireless Commun. and Networking Conf. (WCNC), Apr. 2013, pp. 2902-2907.

[68] A. Elzanati, M. Abdelkader, K. Seddik, and A. Ghuniem, "Adaptive spectrum hole detection using sequential compressive sensing," in Int. Wireless Commun. and Mobile Computing Conf. (IWCMC), Aug. 2014, pp. 1081-1086.

[69] Y. Wang, A. Pandharipande, Y. Polo, and G. Leus, "Distributed compressive wide-band spectrum sensing," in Info. Theory and Applications Workshop, Feb. 2009 , pp. 178-183.

[70] P. Yenduri and A. Gilbert, "Compressive, collaborative spectrum sensing for wideband cognitive radios," in Int. Symp. Wireless Commun. Systems (ISWCS), Aug. 2012, pp. 531-535.

[71] D. Ariananda and G. Leus, "Cooperative compressive wideband power spectrum sensing," in Conference Record of the Forty Sixth Asilomar Conf. on Signals, Systems and Computers (ASILOMAR), Nov. 2012, pp. 303-307.

[72] G. Leus and D. Ariananda, "Power spectrum blind sampling," IEEE Signal Process. Letters, vol. 18, no. 8, pp. 443-446, Aug. 2011.
[73] D. Ariananda and G. Leus, "Compressive wideband power spectrum estimation," IEEE Trans. Signal Process., vol. 60, no. 9, pp. 47754789, Sept. 2012.

[74] Q. Liang, and M. Liu, "Channel Estimation for Opportunistic Spectrum Access: Uniform and Random Sensing”, 2010, availabile online: arXiv:1005.2544 [cs.IT].

[75] T. Mathews and Z. Han, "USRP2 implementation of compressive sensing based channel estimation in OFDM," in Proc. Third Int. Conf. on Commun. and Info. Technol., June 2013, pp. 83-87.

[76] Chenhao Qi, Guosen Yue, Lenan Wu, and A. Nallanathan, "Pilot Design for Sparse Channel Estimation in OFDM-Based Cognitive Radio Systems," IEEE Transactions on Vehicular Technology, vol. 63, no. 2, pp. 982-987, Feb. 2014.

[77] Hanqing Li, Qing Guo, Tao Tang, and Qingzhong Li, "Distributed Resource Allocation for Cognitive Radio Network with Imperfect Spectrum Sensing," in IEEE Vehicular Technology Conference (VTC Fall), pp. 1-6, 2-5 Sept. 2013.

[78] Min Jia, Xin Liu, and Xuemai Gu, "Channel Estimation Algorithm Based on Compressive Sensing for NC-OFDM Systems in Cognitive Radio Context", in Int. J. Advancements in Computing Technology (IJACT), Vol. 5, No. 1, Jan. 2013.

[79] Liaoyuan Zeng, Sean McGrath, and Guanghui Liu, "Cognitive ultra wideband radio spectrum sensing window length optimization algorithm”, EURASIP J. Wireless Communications and Networking, vol. $18,2014$.

[80] W. U. Bajwa, et al., "Compressed channel sensing," Proc. 42nd Annual Conf. Info. Sciences and Systems, pp. 5-10, Mar. 2008.

[81] J. Paredes, G. Arce, and Z. Wang, "Ultra-wideband compressed sensing: Channel estimation," IEEE J. Sel. Topics in Sig. Process., vol. 1, no. 3, pp. 383-395, Oct. 2007.

[82] E. Lagunas and M. Najar, "Sparse channel estimation based on compressed sensing for ultra wideband systems," IEEE Int. Conf. on Ultra WideBand (ICUWB), Sept. 2011.

[83] H. Duan, "MSM-FOCUSS for distributed compressive sensing and wideband DOA estimation," in Proc. 19th Int. Conf. Digital Signal Process. (DSP), Aug. 2014, pp. 400-403.

[84] V. Cevher, A. Gurbuz, J. McClellan, and R. Chellappa, "Compressive wireless arrays for bearing estimation," in Proc. IEEE Int. Conf. on Acoustics, Speech and Signal Process., pp. 24972500, 2008.

[85] Y. Fu and Z. Tian, "Cramer rao bounds for hybrid ToA/DoA-based location estimation in sensor networks," IEEE Signal Process. Letters, vol. 16 , no. 8, pp. 655-658, 2009

[86] K. Han, Y. Wang, B. Kou, and W. Hong, "Parameters estimation using a random linear array and compressed sensing," in Proc. Int. Congress on Image and Signal Process. (CISP), vol. 8, pp. 3950-3954, 2010.

[87] Z. Tang, G. Blacquiere, and G. Leus, "Aliasing-free wideband beamforming using sparse signal representation," IEEE Trans. Signal Process., vol. 59, no. 7, pp. 3464-3469, July 2011.

[88] M. Hyder and K. Mahata, "Direction-of-arrival estimation using a mixed $\ell_{2,0}$ norm approximation," IEEE Trans. Signal Process., vol. 58, no. 9, pp. 4646-4655, Sept. 2010.

[89] S. K. Sharma, S. Chatzinotas, and B. Ottersten, "SNR estimation for multi-dimensional cognitive receiver under correlated channel/noise," IEEE Trans. Wireless Commun., vol. 12, no. 12, pp. 6392-6405, Dec. 2013.

[90] S. K. Sharma, S. Chatzinotas, and B. Ottersten, "Compressive SNR estimation for wideband cognitive radio under correlated scenarios," in Proc. IEEE Wireless Commun. and Networking Conf. (WCNC), Apr. 2014, pp. 713-718.

[91] S. K. Sharma, S. Chatzinotas, and B. Ottersten, "Compressive sparsity order estimation for wideband cognitive radio receiver," IEEE Trans. Signal Process., vol. 62, no. 19, pp. 4984-4996, Oct. 2014.

[92] C. Feng, S. Valaee, and Z. Tan, "Multiple target localization using compressive sensing," in Proc. IEEE Global Telecommun. Conf., Nov. 2009, pp. 1-6.

[93] H. Shi, R. V. Prasad, and I. G. M. M. Niemegeers, "An intra-cell peer to peer protocol in IEEE 802.22 networks," in Proc. IEEE Global Workshops, pp. 533-537, Dec. 2011.

[94] H. Shi, R. V. Prasad, V. S. Rao, and I. G. M. M. Niemegeers, "Procedure to build interference map in peer to peer IEEE 802.22 networks," in Proc. IEEE Global Commun. Conf., pp. 1103-1108, Dec. 2012.

[95] Sung-Yin Shih, and Kwang-Cheng Chen, "Compressed Sensing Construction of Spectrum Map for Routing in Cognitive Radio Networks", in Proc. IEEE Vehicular Technology Conference (VTC Spring), pp.1-5, May 2011. 
[96] B. Jayawickrama, E. Dutkiewicz, I. Oppermann, G. Fang, and J. Ding, "Improved performance of spectrum cartography based on compressive sensing in cognitive radio networks," in Proc. IEEE Int. Conf. Commun. (ICC), June 2013, pp. 5657-5661.

[97] H. Jamali-Rad, H. Ramezani, and G. Leus, "Sparse multi-target localization using cooperative access points," in Proc. IEEE Sensor Array and Multichannel Signal Process. Workshop (SAM), June 2012, pp. 353-356.

[98] J. Connor, T. Green, A. Jovancevic, J. Koss, R. Krishnan, M. Norko, W. Ogle, and J. Weinfield, "Scalable spectrum situational awareness using devices of opportunity," in Proc. IEEE Military Commun. Conf. 2012, Oct. 2012, pp. 1-5.

[99] B. Jayawickrama, E. Dutkiewicz, I. Oppermann, and M. Mueck, "Iteratively reweighted compressive sensing based algorithm for spectrum cartography in cognitive radio networks," in Proc. IEEE Wireless Commun. and Networking Conf. (WCNC), Apr. 2014, pp. 719-724.

[100] M.-H. Yoon, Y. Shin, H.-K. Ryu, and J.-M. Woo, "Ultra-wideband loop antenna," Electronics Letters, vol. 46, no. 18, pp. 1249-1251, Sept. 2010.

[101] Z.-C. Hao and J.-S. Hong, "Highly selective ultra wideband bandpass filters with quasi-elliptic function response," IET Microwaves, Antennas Propag., vol. 5, no. 9, pp. 1103-1108, June 2011.

[102] R. Walden, "Analog-to-digital converter survey and analysis," IEEE J. Sel. Areas in Commun., vol. 17, no. 4, pp. 539 -550, Apr. 1999.

[103] M. Ariaudo and et al, "Green radio despite Dirty RF front-end," EURASIP J. on Wireless Commun. and Networking, vol. 146, Apr. 2012.

[104] H. Sun, A. Nallanathan, C.-X. Wang, and Y. Chen, "Wideband spectrum sensing for cognitive radio networks: a survey," IEEE Wireless Commun., vol. 20, no. 2, pp. 74-81, Apr. 2013.

[105] M. Kim and J. Takada, "Efficient Multichannel Wideband Spectrum Sensing Technique Using Filter Bank," Proc. IEEE Int. Symp. Pers. Indoor Mobile Radio Commun. (PIMRC), pp. 1014-1018, Sept. 2009.

[106] H. Sun, W.-Y. Chiu, J. Jiang, A. Nallanathan, and H. Poor, "Wideband spectrum sensing with sub-Nyquist sampling in cognitive radios," IEEE Trans. Signal Process., vol. 60, no. 11, pp. 6068 -6073, Nov. 2012.

[107] D. Cabric and R. Brodersen, "Physical layer design issues unique to cognitive radio systems," in Proc. Int. Symp. Personal, Indoor and Mobile Radio Commun., vol. 2, Sept. 2005, pp. 759 -763 Vol. 2

[108] J. Laska, S. Kirolos, M. Duarte, T. Ragheb, R. Baraniuk, and Y. Massoud, "Theory and implementation of an analog-to-information converter using random demodulation," in Proc. IEEE Int. Symp. Circuits and Systems, May 2007, pp. 1959-1962.

[109] J. Laska and et al, "Compressive sensing for dynamic spectrum access networks: Techniques and tradeoffs," in Proc. IEEE Symp. New Frontiers in DySPAN, May 2011, pp. 156-163.

[110] D. Malioutov, S. Sanghavi, and A. Willsky, "Compressed sensing with sequential observations," in Proc. IEEE Int. Conf. Acoustics, Speech and Signal Process., Apr. 2008, pp. 3357 -3360.

[111] M. Mishali, Y. Eldar, O. Dounaevsky, and E. Shoshan, "Xampling: Analog to Digital at sub-Nyquist Rates," IET Cir. Dev. and Systems, vol. 5, no. 1, pp. 8-20, Jan. 2011.

[112] M. Mishali and Y. Eldar, "Blind MultiBand Signal Reconstruction: Compressed Sensing for Analog Signals," IEEE Trans. Signal Proces., vol. 57, no. 3, pp. 993-1009, 2009.

[113] C. Yen and X. Tsai, Y. Wang, "Wideband Spectrum Sensing Based on Sub-Nyquist Sampling," IEEE Trans. Signal Process., vol. 61, no. 12, pp. 3028-3040, June 2013.

[114] M. A. Lexa, M. E. Davies, J. S. Thompson, and J. Nikolic, "Compressive Power Spectral Density Estimation," in Proc. Inter. Conf. on Acoustics, Speech and Sig. Process. (ICASSP), pp. 3884-3887, May 2011.

[115] D. Ariananda and G. Leus, "Compressive Wideband Power Spectrum Estimation,” IEEE Trans. Signal Process., pp. 4775-4788, Sept. 2012.

[116] Z. Quan, W. Zhang, S. Shellhammer, and A. Sayed, "Optimal Spectral Feature Detection for Spectrum Sensing at Very Low SNR," IEEE Trans. Commun., vol. 59, pp. 201-212, Jan. 2011.

[117] E. Lagunas and M. Najar, "Spectral Feature Detection with sub-Nyquist Sampling for Wideband Spectrum Sensing," IEEE Trans. Wireless Commun., Mar. 2015.

[118] E. Lagunas and M. Najar, "Sparse Correlation Matching-Based Spectrum Sensing for Open Spectrum Communications", EURASIP J. Advances in Signal Process., vol. 31, Feb. 2012.

[119] H. Sun, W.-Y. Chiu, and A. Nallanathan, "Adaptive compressive spectrum sensing for wideband cognitive radios," IEEE Commun. Letters, vol. 16 , no. 11 , pp. $1812-1815$, Nov. 2012.
[120] H. Chen X. Chen and W. Meng, "Cooperative communications for cognitive radio networks-from theory to applications," IEEE Comm. Surveys \& Tutorials, vol. 16, no. 3, pp. 1180-1192, 2014.

[121] S. K. Sharma, S. Chatzinotas, and B. Ottersten, "Cooperative spectrum sensing for heterogeneous sensor networks using multiple decision statistics," in Proc. 10th Int. Conf. Cognitive Radio Oriented Wireless Networks (CROWNCOM), Apr. 2015.

[122] M. Eslami, F. Torkamani-Azar, and E. Mehrshahi, "A Centralized PSD Map Construction by Distributed Compressive Sensing," in IEEE Communications Letters, vol. 19, no. 3, pp. 355-358, March 2015.

[123] S. Althunibat, R. Palacios, and F. Granelli, "Performance Optimisation of Soft and Hard Spectrum Sensing Schemes in Cognitive Radio," IEEE Communications Letters, vol. 16, no. 7, pp. 998-1001, July 2012.

[124] Z. Tian, "Compressed wideband sensing in cooperative cognitive radio networks," in Proc. IEEE Global Telecommun. Conf., Nov. 2008, pp. $1-5$.

[125] J. Halamek and et al, "Dynamic range and acquisition system," Measurement Science Review, vol. 1, no. 1, pp. 71-74, 2001.

[126] J. R. Treichler, M. A. Davenport, J. N. Laska, and R. G. Baraniuk, "Dynamic range and compressive sensing acquisition receivers," in Defense Applications of Signal Process. (DASP), July 2011, pp. 1-7.

[127] Y. Wang, Z. Tian, and C. Feng, "Sparsity order estimation and its application in compressive spectrum sensing for cognitive radios," IEEE Trans. Wireless Commun., vol. 11, no. 6, pp. 2116 -2125, June 2012.

[128] E. Candes and B. Recht, "Exact low-rank matrix completion via convex optimization," in Proc. 46th Annual Allerton Conf. on Communication, Control, and Computing, Sept. 2008, pp. 806-812.

[129] J. Meng, W. Yin, H. Li, E. Houssain, and Z. Han, "Collaborative spectrum sensing from sparse observations using matrix completion for cognitive radio networks," in Proc. IEEE Int. Conf. ICASSP, pp. 3114-3117, Mar. 2010

[130] M. Duarte, S. Sarvotham, D. Baron, M. Wakin, and R. Baraniuk, "Distributed compressed sensing of jointly sparse signals," in Conference Record of the Thirty-Ninth Asilomar Conf. on Signals, Systems and Computers, Oct. 2005, pp. 1537-1541.

[131] J. Romberg, "Compressive sensing by random convolution," SIAM J. Imag. Sci., vol. 2, no. 4, pp. 1098-1128, 2009.

[132] J. Romberg, "Sensing by Random Convolution," in Proc. IEEE Int. Workshop on Computational Advances in Multi-Sensor Adaptive Process., pp. 137-140, Dec. 2007.

[133] P. Feng, "Universal Minimum-Rate Sampling and Spectrum-Blind Reconstruction for Multiband Signals," PhD, University of Illinois at Urbana-Champaign, USA, 1997.

[134] M. E. Dominguez-Jitnenez, and N. Gonzalez-Prelcic, "Analysis and design of multirate synchronous sampling schemes for sparse multiband signals," Proc. European Signal Process. Conf. (EUSIPCO), pp.11841188, Aug. 2012.

[135] T. Moon, et al., "Low-cost high-speed pseudo-random bit sequence characterization using nonuniform periodic sampling in the presence of noise," Proc. IEEE VLSI Test Symposium (VTS), pp. 146-151, Apr. 2012.

[136] R. Venkataramani and Y. Bresler, "Optimal Sub-Nyquist Nonuniform Sampling and Reconstruction for Multiband Signals," IEEE Signal Process. Letters, vol. 18, no. 8, pp. 443-446, Aug. 2001.

[137] B. Aziz, S. Traore, A. Nafkha, and D. Le Guennec, "Spectrum sensing for cognitive radio using multicoset sampling," in Proc. IEEE Global Commun. Conf. (GLOBECOM), Dec. 2014, pp. 816-821.

[138] M. Lexa, M. Davies, and J. Thompson, "Reconciling compressive sampling systems for spectrally sparse continuous-time signals," IEEE Trans. Signal Process., vol. 60, no. 1, pp. 155 -171, Jan. 2012.

[139] S. K. Sharma, S. Chatzinotas, and B. Ottersten, "Transmit beamforming for spectral coexistence of satellite and terrestrial networks," in Proc. 8th Int. Conf. Cognitive Radio Oriented Wireless Networks (CROWNCOM), July 2013, pp. 275-281.

[140] S. K. Sharma, S. Chatzinotas, and B. Ottersten, "Interference alignment for spectral coexistence of heterogeneous networks," EURASIP J. Wireless Commun. and Networking, vol. 46, 2013.

[141] S. K. Sharma, S. Chatzinotas, and B. Ottersten, "Cognitive beamhopping for spectral coexistence of multibeam satellites," Int. J. Satellite Commun. and Networking, Mar. 2014.

[142] A. Ijaz, A. Awoseyila, and B. Evans, "Improved snr estimation for BPSK and QPSK signals," Electronics Letters, vol. 45, no. 16, pp. 858-859, July 2009.

[143] M. Fujii and Y. Watanabe, "A study on SNR estimation for cognitive radio," in Proc. IEEE Int. Conf. on Ultra-Wideband (ICUWB), Sept. 2012, pp. $11-15$. 
[144] T. Tian, H. Iwai, and H. Sasaoka, "Pseudo BER based SNRestimation for energy detection scheme in cognitive radio," in Proc. IEEE 75th Veh. Technol. Conference (VTC Spring), May 2012, pp. 1-5.

[145] S. K. Sharma, S. Chatzinotas, and B. Ottersten, "Eigenvalue based sensing and SNR estimation for cognitive radio in presence of noise correlation," Proc. IEEE Trans. Veh. Technol., vol. 62, no. 8, pp. 1-4, 2013.

[146] S. K. Sharma, "Interweave/Underlay Cognitive Radio Techniques and Applications in Satellite Communication Systems", Ph.D. dissertation, University of Luxembourg, Luxembourg, 2014, http://orbilu.uni.lu/ handle/10993/18973.

[147] S. K. Sharma, S. Chatzinotas, and B. Ottersten, "Compressive sparsity order estimation for wideband cognitive radio receiver," in Proc. IEEE Int. Commun. Conf., June 2014.

[148] D. Malioutov, M. Cetin, and A. Willsky, "A sparse signal reconstruction perspective for source localization with sensor arrays," IEEE Trans. Signal Process., vol. 53, no. 8, pp. 3010-3022, Aug. 2005.

[149] J. Riihijarvi, P. Mahonen, M. Petrova, and V. Kolar, "Enhancing cognitive radios with spatial statistics: From radio environment maps to topology engine," in Proc. 4th Int. Conf. CROWNCOM, June 2009, pp. 1-6.

[150] Y. Zhao, L. Morales, J. Gaeddert, K. Bae, J.-S. Um, and J. Reed, "Applying radio environment maps to cognitive wireless regional area networks," in Proc. 2nd IEEE Int. Symp. New Frontiers in Dynamic Spectrum Access Networks, Apr. 2007, pp. 115-118.

[151] R. Mahapatra and E. Strinati, "Interference-aware dynamic spectrum access in cognitive radio network," in Proc. IEEE 22nd Int. Symp. on Personal Indoor and Mobile Radio Commun., Sept. 2011, pp. 396-400.

[152] D. Denkovski, V. Rakovic, M. Pavloski, K. Chomu, V. Atanasovski, and L. Gavrilovska, "Integration of heterogeneous spectrum sensing devices towards accurate REM construction," in Proc. IEEE Wireless Commun. and Networking Conf. (WCNC), Apr. 2012, pp. 798-802.

[153] A. Alaya-Feki, S. Ben Jemaa, B. Sayrac, P. Houze, and E. Moulines, "Informed spectrum usage in cognitive radio networks: Interference cartography," in Proc. IEEE 19th Int. Symp. Personal, Indoor and Mobile Radio Commun. (PIMRC), Sept. 2008, pp. 1-5.

[154] J. Bazerque and G. Giannakis, "Distributed spectrum sensing for cognitive radio networks by exploiting sparsity," IEEE Trans. Signal Process., vol. 58, no. 3, pp. 1847-1862, Mar. 2010.

[155] J. Tropp and A. Gilbert, "Signal recovery from random measurements via orthogonal matching pursuit," IEEE Trans. Info. Th., vol. 53, no. 12, pp. 4655 -4666, Dec. 2007.

[156] J. Meng, H. Li, and Z. Han, "Sparse event detection in wireless sensor networks using compressive sensing," in Proc. 43rd Annual Conf. on Info. Sciences and Systems (CISS), 2009, pp. 181-185.

[157] B. Zhang, X. Cheng, N. Zhang, Y. Cui, Y. Li, and Q. Liang, "Sparse target counting and localization in sensor networks based on compressive sensing," in Proc. IEEE INFOCOM, 2011, pp. 2255-2263.

[158] E. Lagunas, S. K. Sharma, S. Chatzinotas, and B. Ottersten, "Compressive sensing based target counting and localization exploiting joint sparsity", to appear in Proc. IEEE ICASSP, March, 2016.

[159] J. Wang, D. Fang, X. Chen, Z. Yang, T. Xing, and L. Cai, "LCS: Compressive sensing based device-free localization for multiple targets in sensor networks," in Proc. IEEE INFOCOM, 2013, pp. 145-149.

[160] M. Weiss, "Passive WLAN radar network using compressed sensing," in IET Int. Conf. Radar Systems, 2012, pp. 1-6.

[161] M. WeiB, "Target detection with a distributed WLAN radar network using compressive sensing," in Proc. 1st Int. Workshop on Compresed Sensing applied to Radar, 2012.

[162] J. Picard and A. Weiss, "Localization of multiple emitters by spatial sparsity methods in the presence of fading channels," in Proc. 7th Workshop on Positioning Navigation and Commun., 2010, pp. 62-67.

[163] V. Cevher, M. F. Duarte, and R. G. Baraniuk, "Distributed target localization via spatial sparsity," in Proc. EUSIPCO, 2008, pp. 25-29.

[164] C. Feng, S. Valaee, and Z. Tan, "Multiple target localization using compressive sensing," in Proc. IEEE Conf. Global Telecommun., 2009, pp. 4356-4361.

[165] T. Do, L. Gan, N. Nguyen, and T. Tran, "Fast and efficient compressive sensing using structurally random matrices," IEEE Trans. Signal Process., vol. 60, no. 1, pp. 139 -154, Jan. 2012.

[166] G. Hattab, and M. Ibnkahla, "Multiband Spectrum Access: Great Promises for Future Cognitive Radio Networks," in Proceedings of the IEEE, vol. 102, no. 3, pp. 282-306, March 2014.

[167] S. K. Sharma, S. Chatzinotas, and B. Ottersten, "3D Beamforming for Spectral Coexistence of Satellite and Terrestrial Networks," in Proc. IEEE Veh. Technol. Conf., Sept. 2015.
[168] E. Lagunas, and M. Najar, "Compressed spectrum sensing in the presence of interference: Comparison of sparse recovery strategies," in Proc. European Signal Process. Conf., pp.1721-1725, 1-5 Sept. 2014.

[169] K. Cao, and H. Shao, "Compressive wideband spectrum sensing using high-order statistics for cognitive radio," in IEEE Global High Tech Congress on Electronics (GHTCE), pp.187-190, 17-19 Nov. 2013.

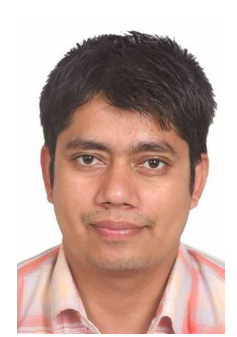

Shree Krishna Sharma (S'12-M'15) received the M.Sc. degree in information and communication engineering from the Institute of Engineering, Pulchowk, Nepal, in 2010; the M.A. degree in economics from Tribhuvan University, Nepal; the M.Res. degree in computing science from Staffordshire University, Staffordshire, U.K., in 2011; and the Ph.D. degree in Wireless Communications from University of Luxembourg, Luxembourg in 2014. Since November 2014, he has been working as a Research Associate in Interdisciplinary Centre for Security, Reliability and Trust (SnT), University of Luxembourg, Luxembourg.

In the past, Dr. Sharma was also involved with Kathmandu University, Dhulikhel, Nepal, as a Teaching Assistant, and he worked as a Part-Time Lecturer for eight engineering colleges in Nepal. He was with Nepal Telecom for more than four years as a Telecom Engineer in the field of information technology and telecommunication. He is the author of more than 50 technical papers in refereed international journals, scientific books, and conferences. He received an Indian Embassy Scholarship for his B.E. study, an Erasmus Mundus Scholarship for his M. Res. study, and an AFR Ph.D. grant from the National Research Fund (FNR) of Luxembourg. He received Best Paper Award in CROWNCOM 2015 conference held in Doha, Qatar and for his Ph.D. thesis, he received FNR award for outstanding PhD Thesis 2015 from FNR, Luxembourg. He has been involved in EU FP7 CoRaSat project, EU H2020 project SANSA, ESA project ASPIM, and Luxembourgish national projects Co2Sat, and SeMIGod. He has been serving as a reviewer for several international journals and conferences, and also as a TPC member for a number of conferences. His research interests include cognitive wireless communications, satellite communications, and signal processing techniques for $5 \mathrm{G}$ and beyond wireless.

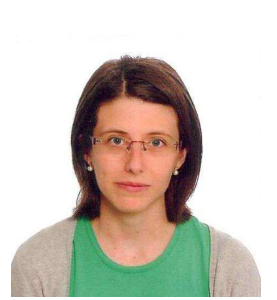

Eva Lagunas (S'09-M'13) received the M.Sc. and $\mathrm{Ph} . \mathrm{D}$. degrees in telecommunications engineering from the Polytechnic University of Catalonia (UPC), Barcelona, Spain, in 2010 and 2014, respectively. She was Research Assistant within the Department of Signal Theory and Communications, UPC, from 2009 to 2013. During the summer of 2009 she was a guest research assistant within the Department of Information Engineering, Pisa, Italy, developing TOA estimation algorithms in UWB systems. From November 2011 to May 2012 she held a visiting research appointment at the Center for Advanced Communications (CAC), Villanova University, PA, USA, where she worked on through-wall radar imaging technology. In September 2014, she joined the Interdisciplinary Centre for Security, Reliability and Trust (SnT), University of Luxembourg, as a Research Associate, where she works on hybrid terrestrial-satellite communications. Dr. Lagunas is an associate editor of EURASIP Journal on Advances in Signal Processing (JASP) since 2015. 


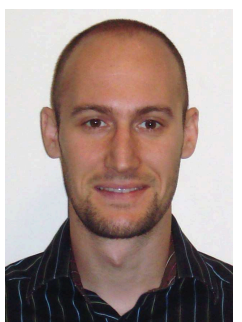

Symeon Chatzinotas (S'06-M'09-SM'13) received the M.Eng. in Telecommunications from Aristotle University of Thessaloniki, Greece and the M.Sc. and Ph.D. in Electronic Engineering from University of Surrey, UK in 2003, 2006 and 2009 respectively. $\mathrm{He}$ is currently a Research Scientist with the research group SIGCOM in the Interdisciplinary Centre for Security, Reliability and Trust, University of Luxembourg, managing H2020, ESA and FNR projects.

In the past, he has worked in numerous RD projects for the Institute of Informatics Telecommunications, National Center for Scientific Research Demokritos, the Institute of Telematics and Informatics, Center of Research and Technology Hellas and Mobile Communications Research Group, Center of Communication Systems Research, University of Surrey. He has authored more than 120 technical papers in refereed international journals, conferences and scientific books. His research interests are on multiuser information theory, cooperative/cognitive communications and wireless networks optimization. Dr Chatzinotas is the corecipient of the 2014 Distinguished Contributions to Satellite Communications Award, Satellite and Space Communications Technical Committee, IEEE Communications Society and CROWNCOM 2015 Best Paper Award. $\mathrm{He}$ is one of the editors of a book on "Cooperative and Cognitive Satellite Systems" published in 2015 by Elsevier and was involved in coorganizing the First International Workshop on Cognitive Radios and Networks for Spectrum Coexistence of Satellite and Terrestrial Systems (CogRaN-Sat) in conjunction with the IEEE ICC 2015, 8-12 June 2015, London, UK.

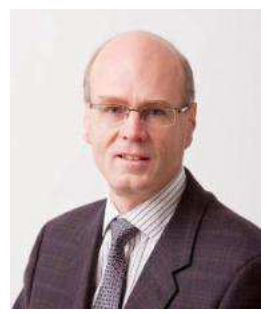

Björn Ottesten (S'87-M'89-SM'99-F'04) was born in Stockholm, Sweden, in 1961. He received the M.S. degree in electrical engineering and applied physics from Linköping University, Linköping, Sweden, in 1986 and the Ph.D. degree in electrical engineering from Stanford University, Stanford, CA, in 1989.

Dr. Ottersten has held research positions at the Department of Electrical Engineering, Linköping University, the Information Systems Laboratory, Stanford University, the Katholieke Universiteit Leuven, Leuven, and the University of Luxembourg. During 96/97, he was Director of Research at ArrayComm Inc, a start-up in San Jose, California based on Ottersten's patented technology. He has co-authored journal papers that received the IEEE Signal Processing Society Best Paper Award in 1993, 2001, 2006, and 2013 and 3 IEEE conference papers receiving Best Paper Awards. In 1991, he was appointed Professor of Signal Processing at the Royal Institute of Technology (KTH), Stockholm. From 1992 to 2004, he was head of the department for Signals, Sensors, and Systems at KTH and from 2004 to 2008, he was dean of the School of Electrical Engineering at KTH. Currently, he is Director for the Interdisciplinary Centre for Security, Reliability and Trust at the University of Luxembourg. As Digital Champion of Luxembourg, he acts as an adviser to European Commissioner Neelie Kroes.

Dr. Ottersten has served as Associate Editor for the IEEE TRANSACTIONS ON SIGNAL PROCESSING and on the editorial board of IEEE Signal Processing Magazine. He is currently editor in chief of EURASIP Signal Processing Journal and a member of the editorial boards of EURASIP Journal of Applied Signal Processing and Foundations and Trends in Signal Processing. He is a Fellow of the IEEE and EURASIP and a member of the IEEE Signal Processing Society Board of Governors. In 2011, he received the IEEE Signal Processing Society Technical Achievement Award. He is a first recipient of the European Research Council advanced research grant. His research interests include security and trust, reliable wireless communications, and statistical signal processing. 